\title{
A new approach for scheduling of multipurpose batch processes with unlimited intermediate storage policy
}

\author{
Nikolaos Rakovitis ${ }^{1}$, Nan Zhang ${ }^{1}$, Jie Li $(\bowtie)^{1}$, Liping Zhang ${ }^{2}$ \\ 1 Centre for Process Integration, School of Chemical Engineering and Analytical Science, The University of Manchester, Manchester, M13 9PL, UK \\ 2 Department of Industrial Engineering, School of Machinery and Automation, Wuhan University of Science and Technology, Wuhan 430081, China
}

(C) The Author(s) 2019. This article is published with open access at link.springer.com and journal.hep.com.cn

\begin{abstract}
The increasing demand of goods, the high competitiveness in the global marketplace as well as the need to minimize the ecological footprint lead multipurpose batch process industries to seek ways to maximize their productivity with a simultaneous reduction of raw materials and utility consumption and efficient use of processing units. Optimal scheduling of their processes can lead facilities towards this direction. Although a great number of mathematical models have been developed for such scheduling, they may still lead to large model sizes and computational time. In this work, we develop two novel mathematical models using the unit-specific eventbased modelling approach in which consumption and production tasks related to the same states are allowed to take place at the same event points. The computational results demonstrate that both proposed mathematical models reduce the number of event points required. The proposed unit-specific event-based model is the most efficient since it both requires a smaller number of event points and significantly less computational time in most cases especially for those examples which are computationally expensive from existing models.
\end{abstract}

Keywords scheduling, multipurpose batch processes, simultaneous transfer, mixed-integer linear programming

\section{Introduction}

Nowadays, it is more important than ever for multipurpose batch process industry to maximize their productivity by simultaneously minimize their costs, fuel and raw material consumption and ecological footprint to be able to survive in a highly competitive market. Developing optimal schedules is one of the main tools that multipurpose

Received December 1, 2018; accepted May 9, 2019

E-mail: jie.li-2@manchester.ac.uk batch process industry can utilize to optimize their processes. Although heuristics-based and spreadsheetbased methods are often used to generate schedules, they are restricted to simple batch processes and often produce suboptimal schedules. Mathematical programming especially mixed-integer programming approaches have been received much attention in the past three decades because they can be used for more complicated batch processes and often provide optimal schedules. Before developing mathematical models, it is crucial to well represent the multipurpose batch process. Two representations have been proposed including state-task network and resourcetask network representations. The state-task network representation (STN) is proposed by Kondili et al. [1] in which all materials in the process are represented by states and processing operations in units are treated as tasks. While states are represented with circles (state nodes), tasks are depicted with rectangles (task nodes). The connections between states and task nodes are depicted with arrows. No resources such as processing units, storage tanks, utilities and manpower are demonstrated in the STN representation. Therefore, the resource-task network representation (RTN) is proposed by Pantelides [2] in which resources used by tasks are explicitly included. Based on the STN and RTN representations, several modelling approaches have been proposed for optimal scheduling of multipurpose batch processes resulting in a great number of mathematical models in the last three decades [3-7]. These modelling approaches include discrete-time $[1,8,9]$, and continuous-time modelling approaches. The continuous-time modelling approaches include slot-based [10-12], global event-based [13-15], unit-specific event-based [16-19] and sequence-based modelling approaches [20-22]. The slot-based modelling approaches can be further classified into process-slot $[11,12]$ and unit-slot $[12,23]$ modelling approaches.

In the discrete-time modelling approach, the scheduling horizon is divided into time intervals of uniform or nonuniform lengths, where the start and end times of each 
interval are known and batches, tasks, or activities are assigned to intervals. Mathematical models developed using this modelling approach are often simple and they usually lead to tight mixed-integer linear programming (MILP) relaxation. A batch, task or activity should start or end exactly at the time interval points. The model sizes largely depend on the number of time intervals required. A great number of time intervals are often required to generate exact solutions, leading to computationally intractable model sizes even for small-scale problems since the length of each time interval is equal to the greatest common factor of the processing times of all units. To avoid an intractable number of time intervals required, continuous-time modelling approaches have been proposed in which the scheduling horizon is divided into ordered slots or event points with non-uniform unknown lengths. Batches, tasks, or activities are assigned to slots or event points. A batch, task or activity should start or end exactly at the slot points or event points. The model sizes also largely depend on the number of slots or event points required. The continuous-time modelling approaches require a significantly smaller number of time slots or event points. However, they often lead to worse MILP relaxation than the discrete-time modelling approach mainly due to the fact that they have to introduce a number of big-M terms in sequencing constraints. In the process slot-based and global event-based continuous-time modelling approaches, time slots or event points are common or shared for all processing units in the process. In other words, batches, tasks or activities in all processing units must start or end at the same slots or event points. In the unit-specific event-based and unit-slot modelling approaches, each unit has independent or separate time slots or event points. The same time slots or event points for different units can start or end at different times. Therefore, the unit-specific event-based or unit-slot modelling approaches often require a smaller number of slots or event points compared to the process slot-based and global event-based modelling approaches, leading to smaller model size and less computational time in general. While the unit-specific event-based modelling approach divides the scheduling horizon using event points where the next event point is not necessarily immediately start after its previous event point end, the unit-slot modelling approach divides the scheduling horizon based on slots where the next slot must immediately start after its previous slots end. In general, the unit-slot modelling approach is very similar to the unit-specific event-based modelling approach. Finally, the sequence-based modelling approach employs direct (immediate) or indirect (general) sequencing (precedence) of task-pairs on units to define a schedule. Time is not explicitly modelled in terms of slots or event points. Although it is not necessary for the sequence-based modelling approach to postulate the numbers of slots or event points a priori, they must postulate the number of batches, tasks or activities a priori.
In addition, they do suffer from the difficulty in monitoring resource levels.

The capabilities of the unit-specific event-based modelling approach have been well established in the literature $[17,24,25]$ with fewer number of event points and smaller model size, which often lead to smaller computational expenses. In most mathematical models developed using the unit-specific event-based modelling approach, the timing variables are defined based on tasks, not on units. In other words, the independent or separate event points are used for tasks, not for units. Therefore, we call them as task-specific event-based models in this work. Most of these task-specific event-based models still require a high number of event points to generate optimal schedules, leading to large model sizes and computational time. This is because most of these models do not allow consumption and production tasks related to the same states to take place at the same event points, unnecessarily increasing the number of event points required. Recently, Shaik and Vooradi [26] proposed a task-specific event-based model for scheduling of multipurpose batch processes, allowing production and consumption tasks related to the same states to take place at the same event points. However, their model is only applicable to the batch process without any recycling loop.

In this work, we develop two novel mathematical models using the unit-specific event-based modelling approach in which related consumption and production tasks are allowed to take place at the same event points. While we define timing variables based on units in one model (called unit-specific event-based model), the timing variables are defined based on tasks in the other model, (called task-specific event-based model). Both formulations are developed based on the STN representation. To make our models applicable for any batch processes, we introduce a definition of recycling tasks slightly different than the definition of recycling tasks of $\mathrm{Li}$ et al. [5]. We only allow non-recycling production and consumption tasks to take place at the same event points to avoid suboptimality. The computational results demonstrate that both proposed mathematical models are very general and can be applied for all batch processes even those with recycling loop and reduce the number of event points required. The proposed unit-specific event-based model is the most efficient since it both requires a smaller number of event points and significantly less computational time in most cases especially for those examples which are computationally expensive from existing models.

\section{Problem description}

A general multipurpose batch process facility including $J(j=1,2, \ldots, J)$ processing units such as reactors, separators and heaters. The STN representation of a multipurpose batch process facility is presented in Fig. 1. 
These units are used to produce $P(p=1,2, \ldots, P)$ final products using $F(f=1,2, \ldots, F)$ feeds. $I(i=1,2, \ldots, I)$ tasks will be processed in the processing units. Each processing unit can process $\mathbf{I}_{j}$ tasks. At each time, at most one task can be processed in a processing unit. Besides final products, intermediate states are also produced. There are total $S(s=1,2, \ldots, S)$ states including feeds, intermediate states, and final products. The feeds are denoted as $\mathbf{S}^{\mathrm{R}}$, the intermediate states are denoted as $\mathbf{S}^{\mathrm{IN}}$, and the final products are included in the set of $\mathbf{S}^{\mathrm{FP}}$. The proportion of each state $s$ produced or consumed by a task $i$ in a unit $j$ is denoted by $\rho_{i, j, s}$. While positive values of $\rho_{i, j, s}$ denote production of state $s$ during the processing of task $i$ in unit $j$, negative values of $\rho_{i, j, s}$ denote consumption of state $s$ during the processing of task $i$ in unit $j$. After production, each batch is allowed to be mixed with other batches or split into several batches for further processing. Some intermediate states are also allowed to be recycled back if necessary. Each intermediate state has its dedicated storage. If the storage capacity for an intermediate state is unlimited, then it is called unlimited intermediate storage (UIS) policy. If the storage capacity is limited or finite, then it is called finite intermediate storage (FIS) policy. If there is no intermediate storage, then it is called no intermediate storage (NIS) policy. In this paper, we assume UIS for all states including intermediate states, feeds and final products. After production in a processing unit, an intermediate state may or may not be allowed to remain in this processing unit. If an intermediate state has to be transferred immediately to storage or other processing units after production, it is called zero wait (ZW) policy. If an intermediate state is allowed to remain in a processing unit with unlimited time, then it is called unlimited wait (UW) policy. If an intermediate state is allowed to be held in a processing unit with a certain time, then it is called limited wait (LW) policy. In this paper, we also assume UW policy for all intermediate states. By introducing this, the scheduling problem can be stated as follows, Given: (1) STN representation of a multipurpose batch facility; (2) J units, unit capacities, suitable tasks and their processing times; (3) S states, the portion of states produced or consumed from a task in a processing unit; (4) Product prices; (5) Scheduling horizon. Determine: (1) Optimal production schedule involving task allocations, start and end timings, sequences and batch sizes; (2) Inventory profiles. Operating rules: (1) At most one task can be processed in a processing unit at any time; (2) Batch mixing and splitting is allowed. Assumptions: (1) All parameters are deterministic; (2) The processing time of a task in a processing unit depends on a fixed processing time (denoted as $\alpha_{i j}$ plus a variable process time based on the batch sizes, which is denoted as $\left(\beta_{i j} \cdot b_{i j}\right)$; (3) Unlimited feed materials are available; (4) Unlimited storage policy for all states; (5) Unlimited resources where required are available; (6) Unlimited wait policy for intermediate states.

The objective is to maximize productivity or minimize makespan. The makespan is defined as the time required to produce a specified demand.

\section{Motivating example}

Let consider an example whose STN representation is depicted in Fig. 2. In this example, a raw material S1 is converted into a final product S3 through two tasks (i.e., I1 and I2) in two processing units (J1 and J2). The scheduling horizon is $9 \mathrm{~h}$. The objective is to maximize the productivity of product S3. All relevant data for this example are given in Table 1. We use the mathematical model of Shaik and Floudas [25] to solve this example. We obtain the optimal solution of $500.00 \mathrm{cu}$ using 2 events. The optimal schedule is illustrated in Fig. 3. As seen from Fig. 3, the task I1, processed in unit J1, produces $100.00 \mathrm{cu}$ of $\mathrm{S} 2$ at event point $\mathrm{N} 1$, which is further processed at task

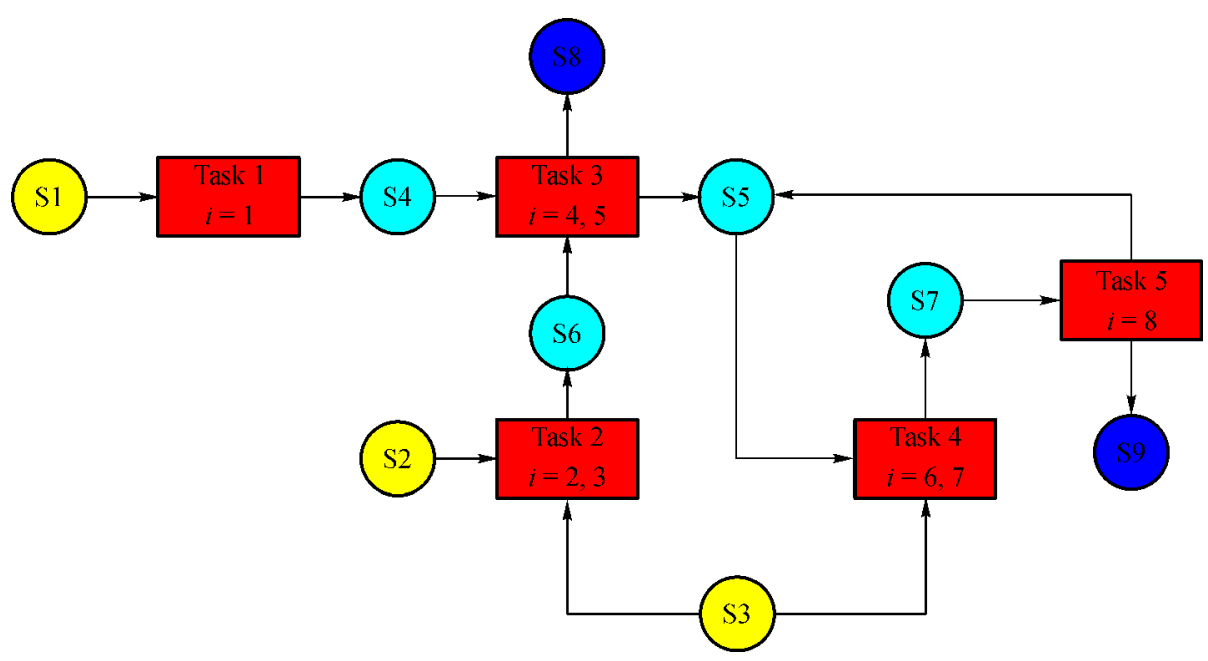

Fig. 1 STN representation of a multipurpose batch process facility (Example 2). 
$\mathrm{I} 2$ in unit $\mathrm{J} 2$ to produce final product $\mathrm{S} 3$ with $100.00 \mathrm{cu}$ at event point N2. This is because the task I2 in unit J2 is a consuming task of S2 and the task $\mathrm{I} 1$ in unit $\mathrm{J} 1$ is a production task for $\mathrm{S} 2$. Therefore, the task 2 must always start at event point $\mathrm{N} 2$ since the production task I1 take place at event point N1 based on the model of Shaik and Floudas [25]. However, we can use one event point for the optimal schedule through analysis. Figure 4 illustrates the optimal schedule with only one event point. From Fig. 4, it can be observed that the consuming task $\mathrm{I} 2$ takes place at the same event point as the production task I1, but not in real time. In real time, task I2 still takes place after I1 is completed. By doing this, we can reduce one event point required for generating the optimal solution. As discussed previously, the model size and computational performance largely depend on the number of event points required. This motivates us to develop new mathematical formulations for scheduling of multipurpose batch facilities by allowing consuming and production tasks related to the same states take place at the same event points to reduce the number of event points required.

\section{Definition of recycling tasks}

Despite the fact that allowing all related production and

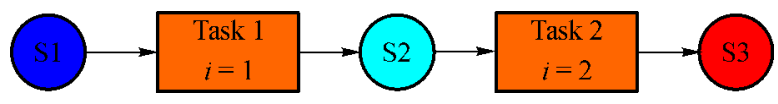

Fig. 2 STN representation of the motivating example.

Table 1 Data for motivating example

\begin{tabular}{lcccc}
\hline Unit & $\begin{array}{c}\text { Maximum } \\
\text { capacity } / \mathrm{mu}\end{array}$ & $\begin{array}{c}\text { Minimum } \\
\text { capacity } / \mathrm{mu}\end{array}$ & $\begin{array}{c}\alpha_{i} \\
/ \mathrm{h}\end{array}$ & $\begin{array}{c}\beta_{i} \\
/ \mathrm{h}\end{array}$ \\
\hline $\mathrm{J} 1$ & 100 & 0 & 3 & 0.02 \\
$\mathrm{~J} 2$ & 100 & 0 & 2 & 0.01 \\
\hline
\end{tabular}

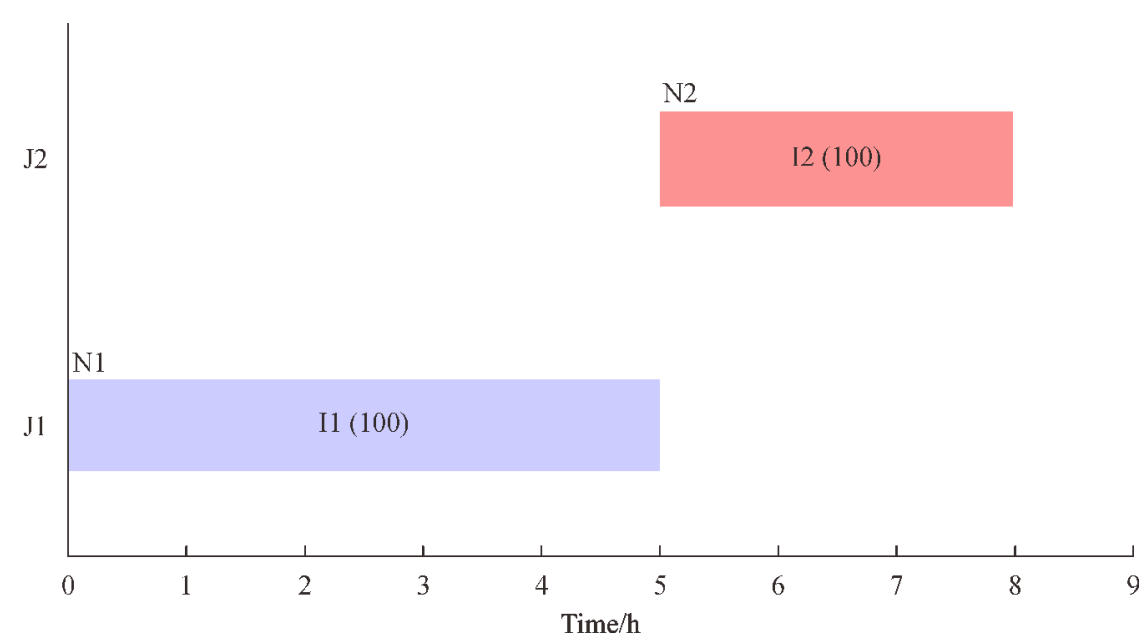

Fig. 3 Optimal schedule for the motivating example using two event points from the model of Shaik and Floudas [25]. consumption tasks take place at the same event points can potentially reduce the number of event points and increase computational efficiency, we could obtain suboptimal solutions in some cases by allowing all production and consumption tasks related to the same states to take place at the same event points. Consider the following example which is depicted in Fig. 5 and Table 2. If production and consumption tasks related to the same states are not allowed to take place at the same event points, the optimal productivity of $1656 \mathrm{cu}$ is generated with four event points from the model of Shaik and Floudas [25]. However, if all production and consumption tasks related to the same states are allowed to take place at the same event points, then the suboptimum productivity of $1511 \mathrm{cu}$ is generated. This occurs from tasks I3, I4 and I5. Note that tasks I4 and I5 produce two states S2 and S4 and task I3 consumes state S2 and produces state S3. If these tasks (i.e., tasks I3, I4, and I5) are allowed to take place at the same event points, it is not possible for these tasks to take place at the same time in real time, which leads to suboptimum solutions.

To avoid suboptimality in such cases, a new definition slightly different from that of recycling tasks of Li et al. [5] is introduced. We define a recycling task in a processing unit if it produces a state that can be consumed either by a task in its upstream processing units or by other tasks in the same processing unit. The recycling tasks are included in the set $\mathbf{I}^{\mathrm{R}}$. In Fig. 6, there are four tasks (I1-I4), two processing units (J1-J2) and three states (S1-S3). While tasks I1 and I 3 can be processed in unit J1, tasks I2 and I4 can be processed in unit $\mathrm{J} 2$. Tasks I1 and I2 consume S1 and produce S2, whilst tasks I3 and I4 consume S2 and produce $\mathrm{S} 1$ and $\mathrm{S} 3$. Based on this new definition, task I1 is considered as a recycling task because it produces S2 that can be used by task I 3 as a raw material in the same unit (i.e., J1). Similarly, tasks I2-I4 are also recycling tasks. Consequently, all tasks in the example depicted in Fig. 6 are considered as recycling tasks. 


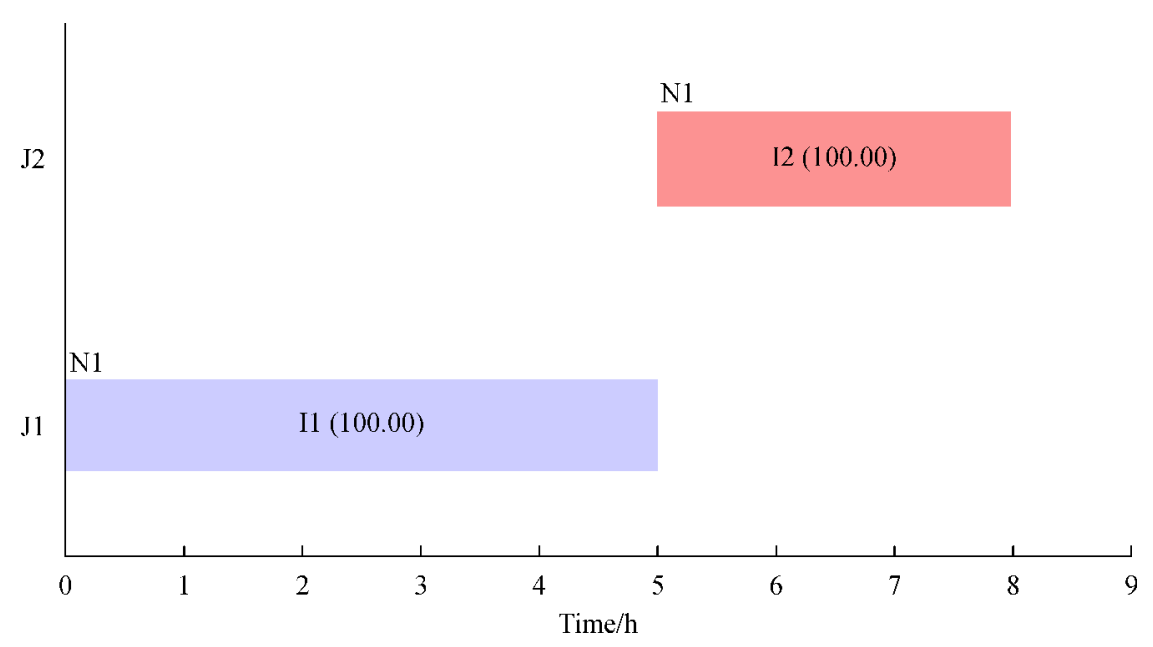

Fig. 4 Optimal schedule for the motivating example using one event point.

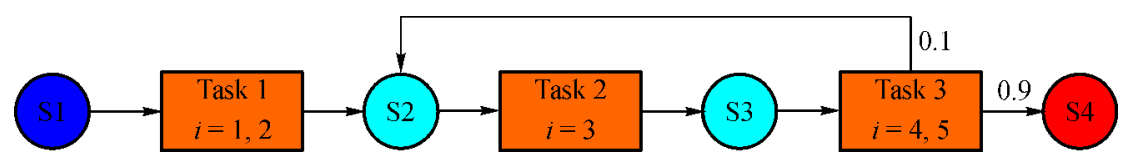

Fig. 5 STN representation of motivating example 2.

Table 2 Results for motivating example $2^{\text {a) }}$

\begin{tabular}{lcccccccc}
\hline Example & Model & $\begin{array}{c}\text { Event } \\
\text { points }\end{array}$ & $\begin{array}{c}\text { CPU time } \\
\text { /s }\end{array}$ & $\begin{array}{c}\text { RMILP } \\
\text { /cu }\end{array}$ & $\begin{array}{c}\text { MILP } \\
\text { /cu }\end{array}$ & $\begin{array}{c}\text { Discrete } \\
\text { variables }\end{array}$ & $\begin{array}{c}\text { Continuous } \\
\text { variables }\end{array}$ & $\begin{array}{c}\text { Equations } \\
\text { 1 }\end{array}$ \\
\hline $\mathrm{H}=8 \mathrm{~h})$ & $\mathrm{SF}$ & 4 & 0.094 & 1800.00 & 1656.16 & 20 & 78 & 115 \\
\hline
\end{tabular}

a) SF: the model of Shaik and Floudas [25]; T-S: the revised model of Shaik and Floudas [25] allowing all production and consumption tasks related to the same states take place at the same event points; CPU: Central processing unit; RMILP: relaxed mixed-integer linear program.

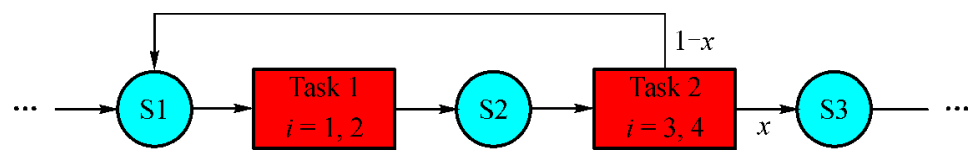

Fig. 6 Illustration of recycling tasks where all tasks are recycling tasks.

\section{Mathematical formulation}

\subsection{Time representation}

As discussed before, the advantages of the unit-specific event-based modelling approach have been well established in the literature. This modelling approach is used to develop our new models, which are presented below.

\subsection{Model M1}

In this model, the timing variables are defined based on units. Therefore, this proposed model is called the unitspecific event-based model.

\subsubsection{Allocation constraints}

To assign tasks to units, we define binary variables $w_{i, j, n, n^{\prime}}$ to denote if a task $i$ is processed in a unit $j$ from event point $n$ to event point $n^{\prime}$. We allow a task in a unit to span over $\Delta n$ event points to make the model general where $\Delta n$ is a parameter that could be used to control the number of event points that a task can span across. At most one task is allowed to take place in a unit during a time as specified by Constraint (1). If tasks are allowed to span over more than one events $(\Delta n>0)$, Constraint (1) allows at most one task to be active from event point $n$ to event point $n^{\prime}$.

$$
\sum_{i \in I_{j}} \sum_{n-\Delta n \leqslant n^{\prime} \leqslant n} \sum_{n \leqslant n^{\prime \prime} \leqslant n^{\prime}+\Delta n} w_{i, j, n^{\prime}, n^{\prime \prime}} \leqslant 1 \quad \forall j, n .
$$




\subsubsection{Capacity constraints}

We define variables $b_{i, j, n, n^{\prime}}$ to denote the amount of materials (i.e., batch size) processed by a task $i$ in a unit $j$ from event point $n$ to event point $n^{\prime}$. If a unit $j$ processes a task $i$ from event point $n$ to event point $n^{\prime}$, then the material processed in this unit should be constrained by the minimum $\left(B_{i, j}^{\min }\right)$ and maximum $\left(B_{i, j}^{\max }\right)$ capacity limits.

$$
\begin{gathered}
B_{i, j}^{\min } w_{i, j, n, n^{\prime}} \leqslant b_{i, j, n, n^{\prime}} \leqslant B_{i, j}^{\max } w_{i, j, n, n^{\prime}} \\
\forall j, i \in \mathbf{I}_{j}, n \leqslant n^{\prime} \leqslant n+\Delta n .
\end{gathered}
$$

\subsubsection{Material balance}

We define $S T_{s, n}$ to denote the amount of material $s$ at event point $n$, which is used to monitor inventory of the materials in storage and ensure no storage capacity violation. Since we allow non-recycling tasks to take place at the same event points as the related consumption tasks, these nonrecycling tasks produce materials at event point $n$. However, recycling tasks have to produce materials at event point $(n-1)$. With this, the amount of material in a storage at an event point $n$ should be equal to the amount of materials at the previous event point $(n-1)$ plus the material produced from non-recycling tasks at event point $n$ and recycling tasks at event point $(n-1)$ minus the material consumed by consumption tasks at event point $n$, as indicated in Constraints (3) and (4).

$$
\begin{aligned}
S T_{s, n}= & S T 0_{s}+\sum_{j} \sum_{i \in\left(\mathbf{I}_{j} \cap \mathbf{I}_{S}^{P}\right), i \notin \mathbf{I}^{\mathrm{R}}} \rho_{i, j, s} \sum_{n-\Delta n \leqslant n^{\prime} \leqslant n} b_{i, j, n^{\prime}, n} \\
& +\sum_{j} \sum_{i \in\left(\mathbf{I}_{j} \cap \mathbf{I}_{S}^{C}\right)} \rho_{i, j, s} \sum_{n \leqslant n^{\prime} \leqslant n+\Delta n} b_{i, j, n, n^{\prime}} \forall s, n=1, \\
S T_{s, n}= & S T_{s, n-1}+\sum_{j} \sum_{i \in\left(\mathbf{I}_{j} \cap \mathbf{I}_{S}^{P}\right), i \notin \mathbf{I}^{\mathrm{R}}} \rho_{i, j, s} \sum_{n-\Delta n \leqslant n^{\prime} \leqslant n} b_{i, j, n^{\prime}, n} \\
& +\sum_{j} \sum_{i \in\left(\mathbf{I}_{j} \cap I_{S}^{P} \cap \mathbf{I}^{R}\right)} \rho_{i, j, s} \sum_{n-1-\Delta n \leqslant n^{\prime} \leqslant n-1} b_{i, j, n^{\prime}, n-1} \\
& +\sum_{j} \sum_{i \in\left(\mathbf{I}_{j} \cap \mathbf{I}_{S}^{C}\right)} \rho_{i, j, s, s} \sum_{n \leqslant n^{\prime} \leqslant n+\Delta n} b_{i, j, n, n^{\prime}} \forall s, n>1 .
\end{aligned}
$$

\subsubsection{Processing duration constraints}

Once a batch is processed on a unit, then it must be processed for some duration. A unit is also allowed to be idle after processing. We define $T_{j, n}^{\mathrm{s}}$ and $T_{j, n}^{\mathrm{f}}$ to denote the start and end times of a processing unit $j$ at event point $n$. The end time of a unit $j$ at event $n$ must be greater than the total processing time, consisting of a fixed term and a variable term depending on the batch size, as indicated in the Constraint (5).

$$
\begin{gathered}
T_{j, n}^{\mathrm{f}} \geqslant T_{j, n}^{\mathrm{s}}+\sum_{i \in \mathbf{I}_{j}} \sum_{n \leqslant n^{\prime} \leqslant n+\Delta n}\left(\alpha_{i j} \cdot w_{i, j, n, n^{\prime}}+\beta_{i j} \cdot b_{i, j, n, n^{\prime}}\right) \\
\forall j, i \in \mathbf{I}_{j}, n \leqslant n^{\prime} \leqslant n+\Delta n .
\end{gathered}
$$

Note that we do not force the finish time to be equal to the start time plus the total processing time to allow materials produced temporally stored in unit or a unit to be idle after processing, which may lead to a smaller number of event points that is required to generate the optimum solution as claimed by $\mathrm{Li}$ and Floudas [17].

\subsubsection{Sequencing constraints}

Same or different tasks in the same unit: An event point $n$ on a processing unit $j$ must always start after its previous event point on the same unit finishes.

$$
T_{j, n+1}^{\mathrm{s}} \geqslant T_{j, n}^{\mathrm{f}} \forall j, n<N .
$$

Different tasks in different units: We need to sequence consuming and production tasks related to the same states where the consuming and production tasks are different tasks in different units. Although a consuming task is allowed to take place at the same event points with its related production tasks which are non-recycling tasks, this consuming task must always start after its related production tasks finish in real time. We introduce a new continuous variable $T_{s, n}$, which denotes the time that state $s$ is available to be consumed at event point $n$. Then we have:

$$
T_{s, n} \leqslant T_{s, n+1} \quad \forall s \in \mathbf{S}^{\mathrm{IN}}, n .
$$

The finish time of unit $j$, which is related with the production of state $s$ should be before the time that state $s$ is available.

$$
\begin{gathered}
T_{s, n} \geqslant T_{j, n}^{\mathrm{f}}-M\left(1-\sum_{i \in\left(\mathbf{I}_{j} \cap \mathbf{I}_{S}^{P}\right)} \sum_{n-\Delta n \leqslant n^{\prime} \leqslant n} w_{i, j, n^{\prime}, n}\right) \\
\forall s \in \mathbf{S}^{\mathrm{IN}}, j, \sum_{i \in \mathbf{I}_{j}} \rho_{s, i}>0, n .
\end{gathered}
$$

The start time of unit $j$ at event point $n$, which is related with the consumption of state $s$ should be after the time that the state is available if it was produced by a non-recycling task $i^{\prime}$. 


$$
\begin{gathered}
T_{s, n} \leqslant T_{j, n}^{\mathrm{s}}+M \cdot\left(1-\sum_{i \in\left(\mathbf{I}_{j} \cap \mathbf{I}_{S}^{C}\right)} \sum_{n \leqslant n^{\prime} \leqslant n+\Delta n} w_{i, j, n, n^{\prime}}\right) \\
\forall s \in \mathbf{S}^{\mathbf{I N}}{ }_{j}, \sum_{j^{\prime}} \sum_{i^{\prime} \in\left(\mathbf{I}_{j^{\prime}} \cap \mathbf{I}_{S}^{P}\right), i \notin \mathbf{I}^{R}} \rho_{s, i^{\prime}}>0, n .
\end{gathered}
$$

If state $s$ is produced by a recycling task $i^{\prime}$, the end time of unit $j$ at event point $n+1$, which is related with the consumption of state $s$ should be after the time that the state is available instead.

$$
\begin{gathered}
T_{s, n} \leqslant T_{j, n+1}^{\mathrm{s}}+M \cdot\left(1-\sum_{i \in\left(\mathbf{I}_{j} \cap \mathbf{I}_{S}^{C}\right)} \sum_{n+1 \leqslant n^{\prime} \leqslant n+1+\Delta n} w_{i, j, n+1, n^{\prime}}\right) \\
\forall s \in \mathbf{S}^{\mathrm{IN}}, j, \sum_{j^{\prime}} \sum_{i^{\prime} \in\left(\mathbf{I}_{j^{\prime}} \cap \mathbf{I}_{S}^{P} \cap \mathbf{I}^{R}\right)} \rho_{s, i^{\prime}}>0, n .
\end{gathered}
$$

\subsubsection{Objectives}

We consider two different objectives. In the first objective, the productivity of a given facility is maximized for a specified scheduling horizon.

$$
z=\sum_{s} p_{s} \sum_{j} \sum_{i \in\left(\mathbf{I}_{j} \cap \mathbf{I}_{S}^{P}\right)} \sum_{n} \sum_{n \leqslant n^{\prime} \leqslant n+\Delta n} \rho_{i, j, s} \cdot b_{i, j, n, n^{\prime}} .
$$

The other objective is to minimize makespan (denoted as $M S$ ), which is considered as following,

$$
M S \geqslant T_{j, n}^{\mathrm{f}} \forall j, n=N .
$$

In the minimization of makespan problem, it should be also ensured that the total demand is satisfied.

$$
\begin{gathered}
S T_{s, n}+\sum_{j} \sum_{i \in\left(I_{S}^{P} \cap I^{R}\right)} \rho_{i, j, s} \sum_{n-\Delta n \leqslant n^{\prime} \leqslant n} b_{i, j, n^{\prime}, n} \geqslant D_{s} \\
\forall s \in \mathbf{S}^{p}, n=N .
\end{gathered}
$$

We complete our model M1 which comprises of Eqs. (1-11) if maximization of productivity is considered as objective and Eqs. (1-10), (12), (13) if minimization of makespan is considered.

\subsection{Model M2}

In this model, the timing variables are defined based on tasks. The same tasks that can be processed in different units have to be divided into two different tasks. We call this model task-specific event-based model. Production and consumption tasks related to the same states are also allowed to take place at the same event points in this model.

\subsubsection{Allocation constraints}

Similar to the model M1, at most one task is allowed to take place at each event point.

$$
\sum_{i \in \mathbf{I}_{j}} \sum_{n-\Delta n \leqslant n^{\prime} \leqslant n} \sum_{n \leqslant n^{\prime \prime} \leqslant n^{\prime}+\Delta n} w_{i, n^{\prime}, n^{\prime \prime}} \leqslant 1 \quad \forall j, n .
$$

\subsubsection{Capacity constraints}

The batch size of task $i$ from event point $n$ to event point $n^{\prime}$ should be constrained by the maximum and minimum capacities if the task is active. Otherwise, it should be equal to zero. This Constraint (15) is the same as that of Shaik and Floudas [25].

$$
\begin{gathered}
B_{i}^{\min } w_{i, n, n^{\prime}} \leqslant b_{i, n, n^{\prime}} \leqslant B_{i}^{\max } w_{i, n, n^{\prime}} \\
\forall j, i \in \mathbf{I}_{j}, n \leqslant n^{\prime} \leqslant n+\Delta n .
\end{gathered}
$$

\subsubsection{Material balance constraints}

Similar to the model M1, we allow materials that are produced by non-recycling tasks to be consumed by their related consumption tasks at the same event point. However, if the materials are produced by recycling tasks, then they have to be consumed by their related consumption tasks at the next event point.

$$
\begin{aligned}
& S T_{s, n}=S T 0_{s}+\sum_{i \in \mathbf{I}_{S}^{P}, i \notin \mathbf{I}^{\mathrm{R}}} \rho_{i, s} \sum_{n-\Delta n \leqslant n^{\prime} \leqslant n} b_{i, n^{\prime}, n} \\
& +\sum_{i \in \mathbf{I}_{S}^{C}} \rho_{i, s} \sum_{n \leqslant n^{\prime} \leqslant n+\Delta n} b_{i, n, n^{\prime}} \forall s, n=1, \\
& S T_{S, n}=S T_{S, n-1}+\sum_{i \in \mathbf{I}_{S}^{P}, i \notin \mathbf{I}^{\mathrm{R}}} \rho_{i, s} \sum_{n-\Delta n \leqslant n^{\prime} \leqslant n} b_{i, n^{\prime}, n} \\
& +\sum_{i \in\left(\mathbf{I}_{S}^{P} \backslash \mathbf{I}^{R}\right)} \rho_{i, s} \sum_{n-1-\Delta n \leqslant n^{\prime} \leqslant n-1} b_{i, n^{\prime}, n-1} \\
& \quad+\sum_{i \in \mathbf{I}_{S}^{C}} \rho_{i, s} \sum_{n \leqslant n^{\prime} \leqslant n+\Delta n} b_{i, n, n^{\prime}} \forall s, n>1 .
\end{aligned}
$$

\subsubsection{Processing duration constraints}

The finish time of a task $i$ should always be greater than the start time of the same task. It should be also greater than the start time of the task plus the total processing time if the task is active. 


$$
\begin{gathered}
T_{i, n^{\prime}}^{\mathrm{f}} \geqslant T_{i, n}^{\mathrm{s}}+a_{i} \cdot w_{i, n, n^{\prime}}+\beta_{i} \cdot b_{i, n, n^{\prime}} \\
\forall i \in \mathbf{I}_{j}, n \leqslant n^{\prime} \leqslant n+\Delta n .
\end{gathered}
$$

\subsubsection{Sequencing constraints}

Same tasks in the same units: A task $i$ taking place at event point $n+1$ should always start after it finishes at event point $n$. This Constraint (19) is the same as those of Shaik and Floudas [25].

$$
T_{i, n+1}^{\mathrm{s}} \geqslant T_{i, n}^{\mathrm{f}} \forall i \in \mathbf{I}_{j}, n .
$$

Different tasks in the same units: A task $i$ taking place at event point $n+1$ should always start after all other tasks that can be processed in the same unit finish at event point $n$.

$$
T_{i, n+1}^{\mathrm{s}} \geqslant T_{i^{\prime}, n}^{\mathrm{f}} \quad \forall j, i \in \mathbf{I}_{j}, i^{\prime} \in \mathbf{I}_{j}, i \quad i^{\prime}, n<N .
$$

Different tasks in different units: Similar to the model M1, two different sets of constraints are introduced based on whether the production task is a recycling or a nonrecycling task.

$$
\begin{gathered}
T_{i, n}^{\mathrm{s}} \geqslant T_{i^{\prime}, n}^{\mathrm{f}}-M\left(1-\sum_{n-\Delta n \leqslant n^{\prime} \leqslant n} w_{i^{\prime}, n^{\prime}, n}\right) \\
\forall j \neq j^{\prime}, i \in\left(\mathbf{I}_{S}^{C} \cap \mathbf{I}_{j}\right), i^{\prime} \in\left(\mathbf{I}_{S}^{P} \cap \mathbf{I}_{j^{\prime}}\right), i^{\prime} \notin \mathbf{I}^{R}, n, \\
T_{i, n+1}^{\mathrm{s}} \geqslant T_{i^{\prime}, n}^{\mathrm{f}}-M\left(1-\sum_{n-\Delta n \leqslant n^{\prime} \leqslant n} w_{i^{\prime}, n^{\prime}, n}\right) \\
\forall j \neq j^{\prime}, i \in\left(\mathbf{I}_{S}^{C} \cap \mathbf{I}_{j}\right), i^{\prime} \in\left(\mathbf{I}_{S}^{P} \cap \mathbf{I}_{j^{\prime}} \cap \mathbf{I}^{R}\right), n<N .
\end{gathered}
$$

\subsubsection{Tightening constraint}

The duration of all tasks performed in a unit $j$ must not exceed the scheduling horizon.

$$
\sum_{i \in \mathbf{I}_{j}} \sum_{n} \sum_{n \leqslant n^{\prime} \leqslant n+\Delta n}\left(\alpha_{i} \cdot w_{i, n, n^{\prime}}+\beta_{i} \cdot b_{i, n, n^{\prime}}\right) \leqslant H \quad \forall j
$$

\subsubsection{Objectives}

Similar to the model M1, two objectives were also considered in this model M2. In the first objective, the productivity of a given facility is maximized for a specified scheduling horizon.

$$
z=\sum_{s} p_{s} \sum_{i \in \mathbf{I}_{S}^{P}} \sum_{n} \sum_{n \leqslant n^{\prime} \leqslant n+\Delta n} \rho_{i, s} b_{i, n, n^{\prime}} .
$$

The second objective is to minimize makespan.

$$
M S \geqslant T_{i, n}^{\mathrm{f}} \forall i, n=N .
$$

Finally, in the case of minimization of makespan, the total demand should be satisfied.

$$
\begin{gathered}
S T_{s, n}+\sum_{i \in\left(I_{S}^{P} \cap I^{R}\right)} \rho_{i, s} \sum_{n-\Delta n \leqslant n^{\prime} \leqslant n} b_{i, n^{\prime}, n} \geqslant D_{s} \\
\forall s \in \mathbf{S}^{p}, n=N .
\end{gathered}
$$

We complete our model M2 which comprises Eqs. (1424 ) if the maximization of productivity is considered as objective and Eqs. (14-23), (25), (26) if the minimization of makespan is considered as objective.

\section{Computational studies}

We solve twelve examples to illustrate the capability of the proposed models M1 and M2. The data for all examples are given in Tables 3-14. The STN representation of these examples are illustrated in Figs. 1 and 7-16. Note that the STN representation of Example 2 is illustrated in Fig. 1. Among these twelve examples, Examples 1-3 and 8-12 are well-established examples from the literature $[1,17,25]$. These twelve examples have varying tasks, units, recipe structures, processing times, and scheduling horizons. All examples are solved to zero optimality gap using CPLEX 12/GAMS 24.6.1. on a desktop computer with Intel ${ }^{\circledR}$ Core $^{\text {TM }}$ i5-2500 $3.3 \mathrm{GHz}$ and 8 GB RAM running Windows 7. The maximum computational time is set as one hour for all examples.

The computational results from M1 and M2 are presented in Tables 15-20. While Tables 15-19 present the results from M1 and M2 with maximization of productivity as the objective, Table 20 presents the results from both models with minimization of makespan as the objective. From Tables 15-20, it can be observed that both M1 and M2 models are able to generate optimum solutions using less number of event points, which leads to smaller model sizes and less computational time. For instance, both M1 and M2 models require two event points less than the model of Shaik and Floudas [25] to generate the optimal solutions in all instances in Example 1 (see Table 15). More

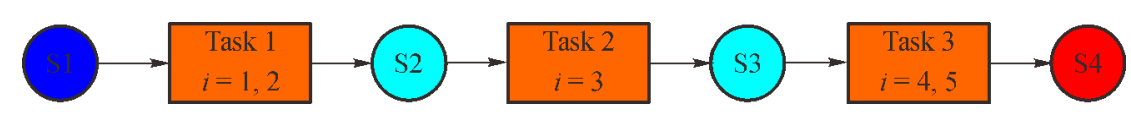

Fig. 7 STN representation of Example 1. 
Table 3 Data for Example 1

\begin{tabular}{lccccc}
\hline Task & Processing unit & $\alpha_{i}$ & $\beta_{i}$ & $B_{i}^{\min }$ & $B_{i}^{\max }$ \\
\hline 1 & 1 & 1.333 & 0.01333 & 0 & 100 \\
2 & 2 & 1.333 & 0.01333 & 0 & 150 \\
3 & 3 & 1.000 & 0.00500 & 0 & 200 \\
4 & 4 & 0.667 & 0.00445 & 0 & 150 \\
5 & 5 & 0.667 & 0.00445 & 0 & 150 \\
\hline
\end{tabular}

Table 4 Data for Example 2

\begin{tabular}{lccccc}
\hline Task & Processing unit & $\alpha_{i}$ & $\beta_{i}$ & $B_{i}^{\min }$ & \\
\hline 1 & 1 & 0.667 & 0.00667 & 0 & 0 \\
2 & 2 & 1.334 & 0.02664 & 0 & 50 \\
3 & 3 & 1.334 & 0.01665 & 0 & 80 \\
4 & 1.334 & 0.02664 & 0 & 50 \\
5 & 2 & 1.334 & 0.01665 & 0 & 80 \\
6 & 3 & 0.667 & 0.01332 & 0 & 80 \\
7 & 2 & 0.667 & 0.008325 & 0 & 200 \\
8 & 3 & 1.334 & 0.00666 & 0 & 80 \\
\hline
\end{tabular}

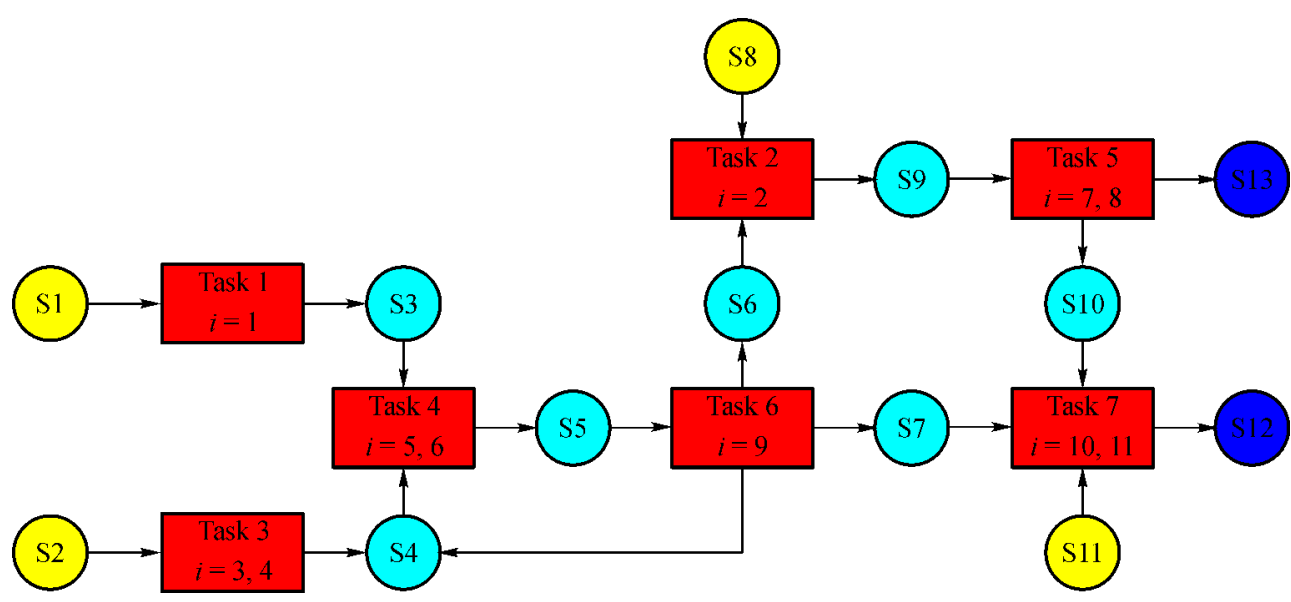

Fig. 8 STN representation of Example 3.

Table 5 Data for Example 3

\begin{tabular}{llllll}
\hline Task & Processing unit & $\alpha_{i}$ & $\beta_{i}$ & $B_{i}^{\min }$ & 0 \\
\hline 1 & 1 & 0.667 & 0.00667 & 0 & 100 \\
2 & 1 & 1.000 & 0.01000 & 0 & 100 \\
3 & 2 & 1.333 & 0.01333 & 0 & 100 \\
4 & 1.333 & 0.00889 & 0 & 150 \\
5 & 3 & 0.667 & 0.00667 & 0 & 100 \\
6 & 2 & 0.667 & 0.00445 & 0 & 100 \\
7 & 3 & 1.333 & 0.01330 & 0 & 150 \\
9 & 2 & 1.333 & 0.00889 & 0 & 300 \\
10 & 3 & 2.000 & 0.00667 & 20 & 200 \\
\hline
\end{tabular}




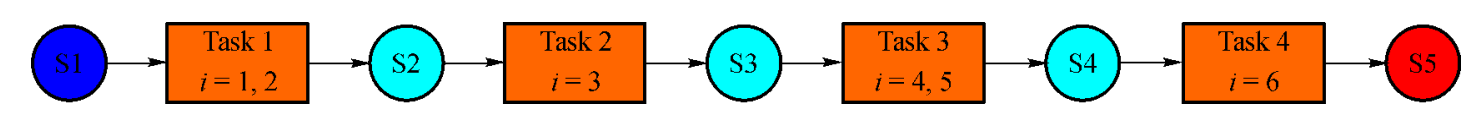

Fig. 9 STN representation of Example 4.

Table 6 Data for Example 4

\begin{tabular}{lccccc}
\hline Task & Processing unit & $\alpha_{i}$ & $\beta_{i}$ & $B_{i}^{\min }$ & $B_{i}^{\max }$ \\
\hline 1 & 1 & 1.333 & 0.01333 & 0 & 100 \\
2 & 2 & 1.333 & 0.01333 & 0 & 150 \\
3 & 3 & 1.000 & 0.00500 & 0 & 200 \\
4 & 4 & 0.667 & 0.00445 & 0 & 150 \\
5 & 5 & 0.667 & 0.00445 & 0 & 150 \\
6 & 6 & 1.000 & 0.00500 & 0 & 200 \\
\hline
\end{tabular}

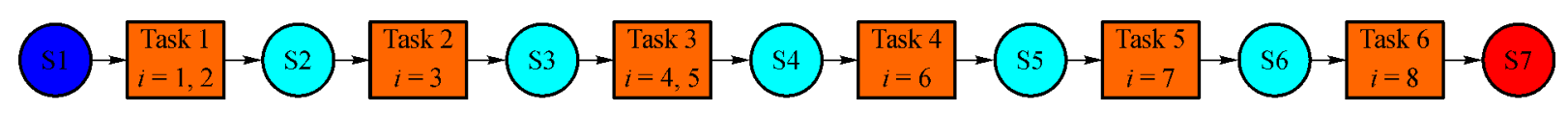

Fig. 10 STN representation of Example 5.

Table 7 Data for Example 5

\begin{tabular}{lccccc}
\hline Task & Processing unit & $\alpha_{i}$ & $\beta_{i}$ & $B_{i}^{\min }$ \\
\hline 1 & 1 & 1.333 & 0.01333 & 0 & 100 \\
2 & 2 & 1.333 & 0.01333 & 0 & 150 \\
3 & 3 & 1.000 & 0.00500 & 0 & 200 \\
4 & 0.667 & 0.00445 & 0 & 150 \\
5 & 5 & 0.667 & 0.00445 & 0 & 150 \\
6 & 6 & 1.000 & 0.00500 & 0 & 100 \\
7 & 7 & 1.333 & 0.01333 & 0 & 100 \\
8 & 8 & 1.333 & 0.01333 & 0 & 150 \\
\hline
\end{tabular}
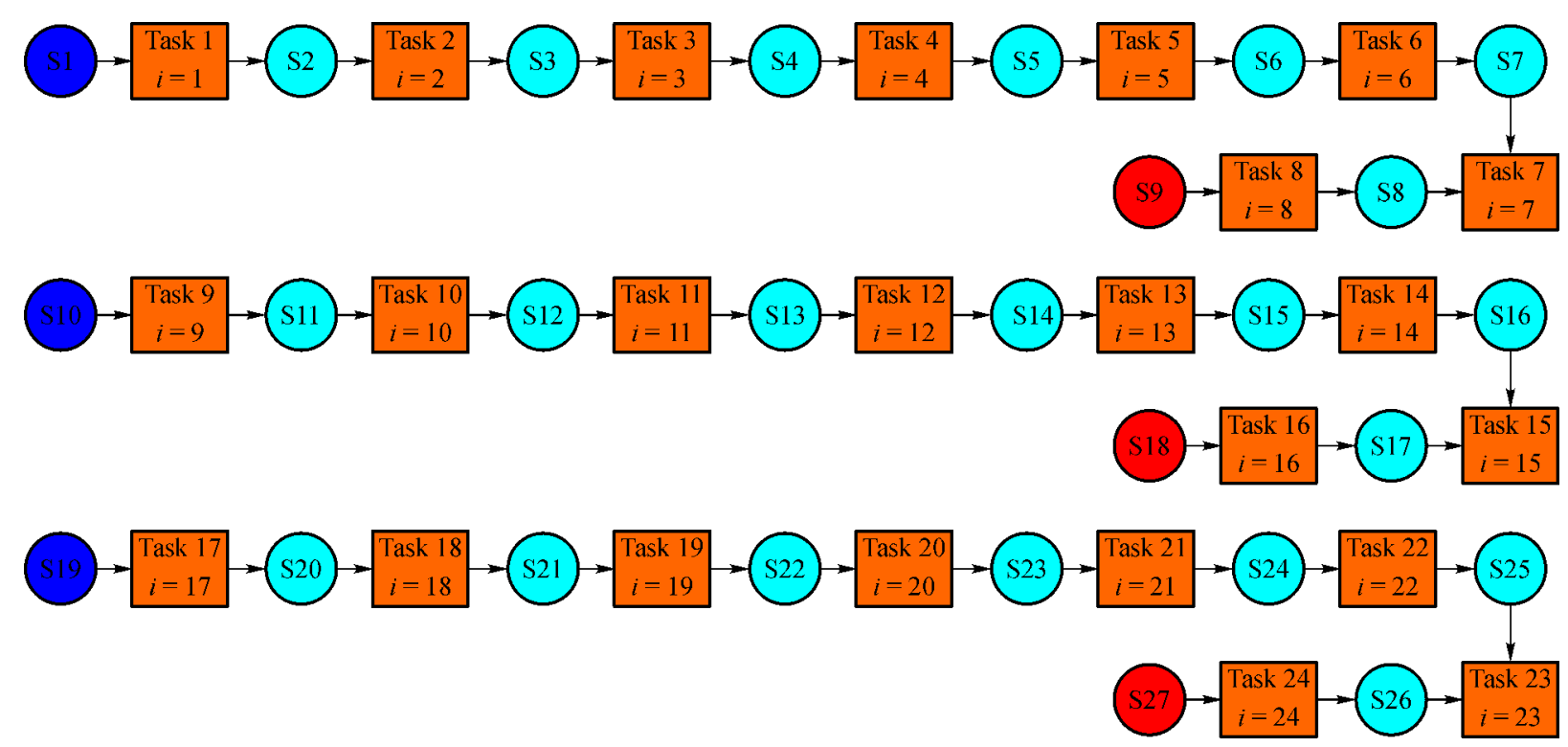

Fig. 11 STN representation of Example 6. 
Table 8 Data for Example 6

\begin{tabular}{lccccc}
\hline Task & Processing unit & $\alpha_{i}$ & $\beta_{i}$ & $B_{i}^{\min }$ & $B_{i}^{\text {max }}$ \\
\hline $1-3$ & 1 & 1.333 & 0.01333 & 0 & 0 \\
$4-6$ & 2 & 1.333 & 0.01333 & 0 & 100 \\
$7-9$ & 3 & 1.000 & 0.00500 & 0 & 150 \\
$10-12$ & 4 & 0.667 & 0.00445 & 0 & 150 \\
$13-15$ & 5 & 0.667 & 0.00445 & 0 & 200 \\
$16-18$ & 6 & 1.000 & 0.00500 & 0 & 100 \\
$19-21$ & 7 & 1.333 & 0.01333 & 0 & 150 \\
$22-24$ & 8 & 1.333 & 0.01333 & 0 \\
\hline
\end{tabular}

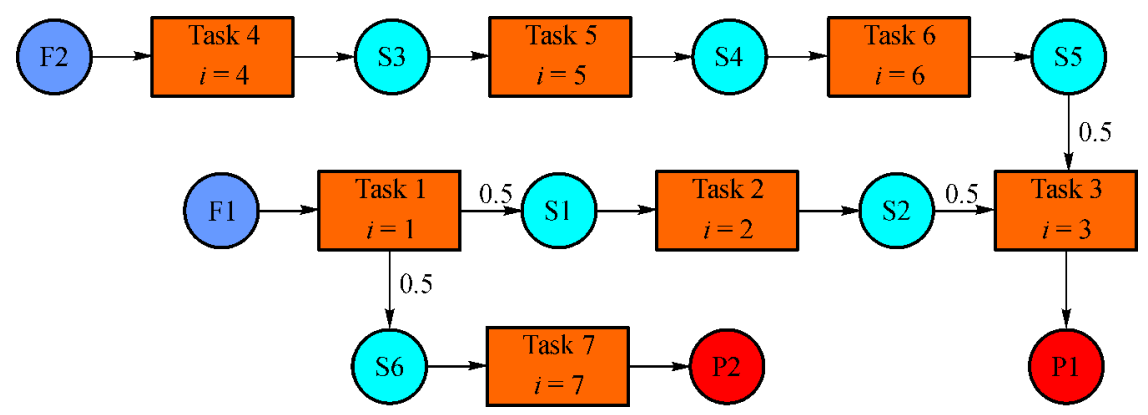

Fig. 12 STN representation of Example 7.

Table 9 Data for Example 7

\begin{tabular}{lccccc}
\hline Task & Processing unit & $\alpha_{i}$ & $\beta_{i}$ & $B_{i}^{\min }$ & $B_{i}^{\max }$ \\
\hline 1 & 1 & 6.000 & 0 & 0 & 0 \\
2 & 2 & 5.000 & 0 & 0 & 100 \\
3 & 3 & 9.000 & 0 & 0 & 100 \\
4 & 4 & 2.000 & 0 & 0 & 50 \\
5 & 5 & 3.000 & 0 & 0 & 50 \\
6 & 6 & 0 & 0 & 50 \\
7 & 7 & 2.000 & 0 & 0 & 100 \\
\hline
\end{tabular}

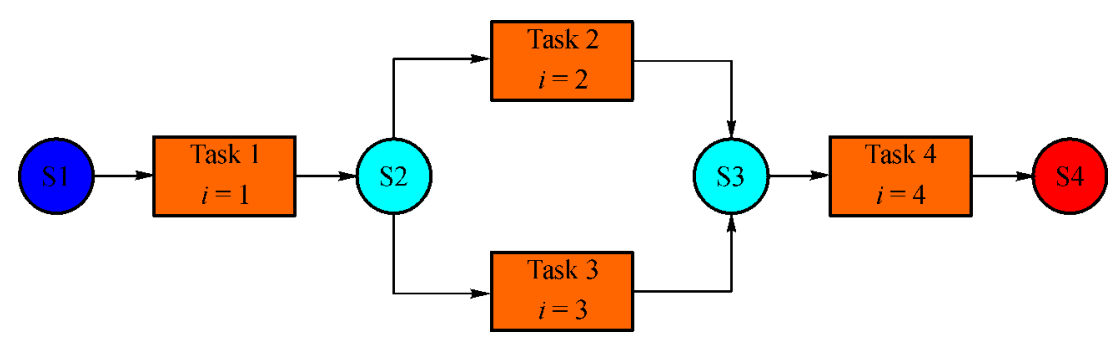

Fig. 13 STN representation of Example 8 .

Table 10 Data for Example 8

\begin{tabular}{lccccc}
\hline Task & Processing unit & $\alpha_{i}$ & $\beta_{i}$ & $B_{i}^{\min }$ & $B_{i}^{\max }$ \\
\hline 1 & 1 & 1.000 & 0 & 0 & 10 \\
2 & 2 & 3.000 & 0 & 0 & 4 \\
3 & 3 & 1.000 & 0 & 0 & 2 \\
4 & 4 & 2.000 & 0 & 0 & 10 \\
\hline
\end{tabular}




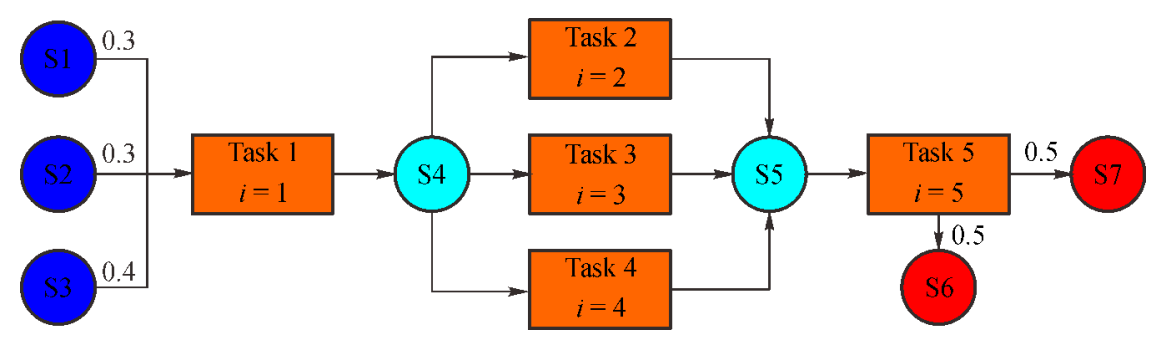

Fig. 14 STN representation of Example 9.

Table 11 Data for Example 9

\begin{tabular}{lccccc}
\hline Task & Processing unit & $\alpha_{i}$ & $\beta_{i}$ & $B_{i}^{\min }$ & $B_{i}^{\max }$ \\
\hline 1 & 1 & 1.500 & 0 & 0 & 0 \\
2 & 2 & 4.500 & 0 & 0 & 60 \\
3 & 3 & 1.500 & 0 & 0 & 30 \\
4 & 4 & 1.500 & 0 & 0 & 30 \\
5 & 5 & 3.000 & 0 & 0 & 150 \\
\hline
\end{tabular}

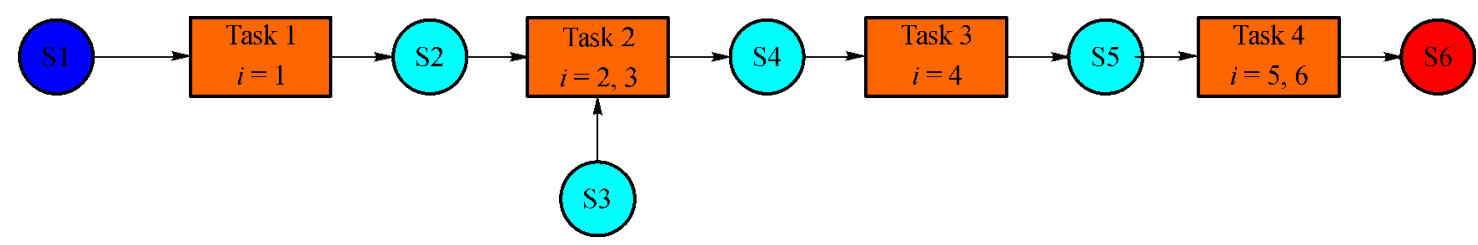

Fig. 15 STN representation of Example 10.

Table 12 Data for Example 10

\begin{tabular}{lccccc}
\hline Task & Processing unit & $\alpha_{i}$ & $\beta_{i}$ & $B_{i}^{\min }$ & 0 \\
\hline 1 & 1 & 17.333 & 0.866 & 0 & 20 \\
2 & 2 & 2.667 & 0.133 & 0 & 20 \\
3 & 3 & 2.667 & 0.133 & 0 & 20 \\
4 & 4 & 0.200 & 0 & 20 \\
5 & 5 & 5.333 & 0.266 & 0 & 20 \\
6 & 6 & 5.333 & 0.266 & 0 & 20 \\
\hline
\end{tabular}

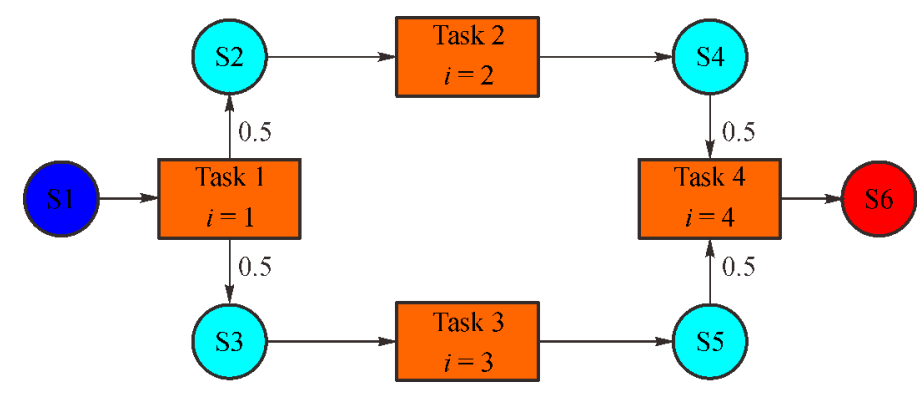

Fig. 16 STN representation of Examples 11 and 12. 
Table 13 Data for Example 11

\begin{tabular}{lccccc}
\hline Task & Processing unit & $\alpha_{i}$ & $\beta_{i}$ & $B_{i}^{\min }$ & $B_{i}^{\max }$ \\
\hline 1 & 1 & 1.666 & 0.03335 & 0 & 40 \\
2 & 2 & 2.333 & 0.08335 & 0 & 20 \\
3 & 3 & 0.667 & 0.06600 & 0 & 5 \\
4 & 4 & 2.667 & 0.008325 & 0 & 40 \\
\hline
\end{tabular}

Table 14 Data for Example 12

\begin{tabular}{lccccc}
\hline Task & Processing unit & $\alpha_{i}$ & $\beta_{i}$ & $B_{i}^{\min }$ & 0 \\
\hline 1 & 1 & 1.666 & 0.03335 & 0 & 40 \\
2 & 2 & 2.333 & 0.08335 & 0 & 20 \\
3 & 3 & 0.333 & 0.06800 & 0 & 2.5 \\
4 & 4 & 2.667 & 0.008325 & 0 & 40 \\
\hline
\end{tabular}

Table 15 Computational results for Example 1 using maximization of productivity as objective ${ }^{\text {a) }}$

\begin{tabular}{|c|c|c|c|c|c|c|c|c|}
\hline Example & Model & $\begin{array}{l}\text { Event } \\
\text { points }\end{array}$ & $\begin{array}{l}\mathrm{CPU} \\
\text { time } / \mathrm{s}\end{array}$ & $\begin{array}{l}\text { RMILP } \\
/ \mathrm{cu}\end{array}$ & $\begin{array}{l}\text { MILP } \\
/ \mathrm{cu}\end{array}$ & $\begin{array}{c}\text { Discrete } \\
\text { variables }\end{array}$ & $\begin{array}{c}\text { Continuous } \\
\text { variables }\end{array}$ & Constraints \\
\hline$\overline{1 a}$ & SF & 4 & 0.078 & 2000.00 & 1840.18 & 20 & 78 & 109 \\
\hline \multirow[t]{2}{*}{$(\mathrm{H}=8 \mathrm{~h})$} & M2 & 2 & 0.125 & 2000.00 & 1840.18 & 10 & 40 & 57 \\
\hline & M1 & 2 & 0.062 & 2000.00 & 1840.18 & 10 & 47 & 58 \\
\hline $1 b$ & $\mathbf{S F}$ & 5 & 0.094 & 3000.00 & 2628.19 & 25 & 97 & 137 \\
\hline \multirow[t]{2}{*}{$(\mathrm{H}=10 \mathrm{~h})$} & M2 & 3 & 0.109 & 3000.00 & 2628.19 & 15 & 59 & 85 \\
\hline & M1 & 3 & 0.047 & 3000.00 & 2628.19 & 15 & 68 & 90 \\
\hline $1 \mathrm{c}$ & $\mathbf{S F}$ & 6 & 0.109 & 4000.00 & 3463.62 & 30 & 116 & 165 \\
\hline \multirow[t]{2}{*}{$(\mathrm{H}=12 \mathrm{~h})$} & M2 & 4 & 0.124 & 4000.00 & 3463.62 & 20 & 78 & 113 \\
\hline & M1 & 4 & 0.078 & 4000.00 & 3463.62 & 20 & 89 & 122 \\
\hline $1 d$ & SF & 9 & 1.29 & 6601.65 & 5038.05 & 45 & 173 & 249 \\
\hline \multirow[t]{2}{*}{$(\mathrm{H}=16 \mathrm{~h})$} & M2 & 7 & 1.54 & 6601.65 & 5038.05 & 35 & 135 & 197 \\
\hline & M1 & 7 & 1.37 & 6601.65 & 5038.05 & 35 & 152 & 218 \\
\hline
\end{tabular}

a) $\Delta n=0$ for all cases

Table 16 Computational results for Example 2 using maximization of productivity as objective

\begin{tabular}{|c|c|c|c|c|c|c|c|c|}
\hline Example & Model & $\begin{array}{l}\text { Event } \\
\text { points }\end{array}$ & $\begin{array}{l}\mathrm{CPU} \text { time } \\
/ \mathrm{s}\end{array}$ & $\begin{array}{l}\text { RMILP } \\
/ \mathrm{cu}\end{array}$ & $\begin{array}{c}\text { MILP } \\
/ \mathrm{cu}\end{array}$ & $\begin{array}{c}\text { Discrete } \\
\text { variables }\end{array}$ & $\begin{array}{c}\text { Continuous } \\
\text { variables }\end{array}$ & Constraints \\
\hline $2 \mathrm{a}$ & SF & $4(\Delta n=0)$ & 0.078 & 1730.87 & 1498.57 & 32 & 136 & 211 \\
\hline \multirow{2}{*}{$(\mathrm{H}=8 \mathrm{~h})$} & M2 & $4(\Delta n=0)$ & 0.141 & 1730.87 & 1498.57 & 32 & 136 & 213 \\
\hline & M1 & $4(\Delta n=0)$ & 0.062 & 1730.87 & 1498.57 & 32 & 126 & 180 \\
\hline $2 b$ & SF & $6(\Delta n=0)$ & 0.889 & 2730.66 & 1943.17 & 48 & 202 & 331 \\
\hline \multirow[t]{5}{*}{$(\mathrm{H}=10 \mathrm{~h})$} & M2 & $6(\Delta n=0)$ & 0.889 & 2730.66 & 1943.17 & 48 & 202 & 331 \\
\hline & M1 & $6(\Delta n=0)$ & 0.827 & 2730.66 & 1943.17 & 48 & 184 & 276 \\
\hline & SF & $6(\Delta n=1)$ & 5.41 & 2730.66 & 1962.69 & 88 & 242 & 737 \\
\hline & M2 & $6(\Delta n=1)$ & 5.10 & 2730.66 & 1962.69 & 88 & 242 & 739 \\
\hline & M1 & $6(\Delta n=1)$ & 2.78 & 2730.66 & 1962.69 & 88 & 224 & 316 \\
\hline $2 c$ & SF & $7(\Delta n=0)$ & 2.39 & 3301.03 & 2658.52 & 56 & 235 & 388 \\
\hline \multirow[t]{2}{*}{$(\mathrm{H}=12 \mathrm{~h})$} & M2 & $7(\Delta n=0)$ & 2.78 & 3301.03 & 2658.52 & 56 & 235 & 390 \\
\hline & M1 & $7(\Delta n=0)$ & 2.86 & 3301.03 & 2658.52 & 56 & 213 & 324 \\
\hline $2 \mathrm{~d}$ & $\mathbf{S F}$ & $8(\Delta n=0)$ & 5.97 & 4291.68 & 3738.38 & 64 & 268 & 447 \\
\hline \multirow[t]{2}{*}{$(\mathrm{H}=16 \mathrm{~h})$} & M2 & $8(\Delta n=0)$ & 6.30 & 4291.68 & 3738.38 & 64 & 268 & 449 \\
\hline & M1 & $8(\Delta n=0)$ & 3.94 & 4291.68 & 3738.38 & 64 & 242 & 372 \\
\hline
\end{tabular}


Table 17 Computational results for Example 3 using maximization of productivity as objective

\begin{tabular}{|c|c|c|c|c|c|c|c|c|}
\hline Example & Model & $\begin{array}{l}\text { Event } \\
\text { points }\end{array}$ & $\begin{array}{c}\mathrm{CPU} \text { time } \\
/ \mathrm{s}\end{array}$ & $\begin{array}{c}\text { RMILP } \\
/ \mathrm{cu}\end{array}$ & $\begin{array}{l}\text { MILP } \\
/ \mathrm{cu}\end{array}$ & $\begin{array}{c}\text { Discrete } \\
\text { variables }\end{array}$ & $\begin{array}{l}\text { Continuous } \\
\text { variables }\end{array}$ & Constraints \\
\hline $3 a$ & SF & $5(\Delta n=0)$ & 0.218 & 2100.00 & 1583.44 & 55 & 235 & 390 \\
\hline \multirow[t]{2}{*}{$(\mathrm{H}=8 \mathrm{~h})$} & M2 & $5(\Delta n=0)$ & 0.343 & 2100.00 & 1583.44 & 55 & 235 & 390 \\
\hline & M1 & $5(\Delta n=0)$ & 0.358 & 2100.00 & 1583.44 & 55 & 229 & 346 \\
\hline $3 b$ & SF & $7(\Delta n=0)$ & 6.24 & 3369.69 & 2305.55 & 77 & 327 & 560 \\
\hline \multirow[t]{5}{*}{$(\mathrm{H}=10 \mathrm{~h})$} & M2 & $7(\Delta n=0)$ & 6.42 & 3369.69 & 2305.55 & 77 & 327 & 560 \\
\hline & M1 & $7(\Delta n=0)$ & 10.23 & 3369.69 & 2293.46 & 77 & 315 & 494 \\
\hline & SF & $8(\Delta n=1)$ & 3159 & 3618.64 & 2358.20 & 165 & 450 & 1433 \\
\hline & M2 & $8(\Delta n=1)$ & 3141 & 3618.64 & 2358.20 & 165 & 450 & 1433 \\
\hline & M1 & $8(\Delta n=1)$ & 892 & 3618.64 & 2358.20 & 165 & 435 & 659 \\
\hline $3 \mathrm{c}$ & SF & $7(\Delta n=0)$ & 0.437 & 3465.63 & 3041.27 & 77 & 327 & 560 \\
\hline \multirow[t]{2}{*}{$(\mathrm{H}=12 \mathrm{~h})$} & M2 & $7(\Delta n=0)$ & 0.406 & 3465.63 & 3041.27 & 77 & 327 & 560 \\
\hline & M1 & $7(\Delta n=0)$ & 0.483 & 3465.63 & 3041.27 & 77 & 315 & 494 \\
\hline $3 d$ & SF & $10(\Delta n=0)$ & 7.80 & 5225.86 & 4262.80 & 110 & 465 & 815 \\
\hline \multirow[t]{2}{*}{$(\mathrm{H}=16 \mathrm{~h})$} & M2 & $10(\Delta n=0)$ & 6.99 & 5225.86 & 4262.80 & 110 & 465 & 715 \\
\hline & M1 & $10(\Delta n=0)$ & 8.81 & 5225.86 & 4262.80 & 110 & 444 & 716 \\
\hline
\end{tabular}

Table 18 Computational results for Examples 4-7 using maximization of productivity as objective ${ }^{\text {a) }}$

\begin{tabular}{|c|c|c|c|c|c|c|c|c|}
\hline Example & Model & Event points & CPU time $/ \mathrm{s}$ & $\begin{array}{l}\text { RMILP } \\
/ \mathrm{cu}\end{array}$ & $\begin{array}{l}\mathrm{MILP} \\
/ \mathrm{cu}\end{array}$ & $\begin{array}{c}\text { Discrete } \\
\text { variables }\end{array}$ & $\begin{array}{c}\text { Continuous } \\
\text { variables }\end{array}$ & Constraints \\
\hline 4 & SF & 10 & 24.18 & 6601.65 & 4305.46 & 60 & 232 & 345 \\
\hline \multirow[t]{2}{*}{$(\mathrm{H}=16 \mathrm{~h})$} & M2 & 7 & 27.63 & 6601.65 & 4305.46 & 42 & 163 & 246 \\
\hline & M1 & 7 & 35.88 & 6601.65 & 4305.46 & 42 & 188 & 279 \\
\hline $5 \mathrm{a}$ & SF & 8 & 0.141 & 1500.00 & 1414.18 & 64 & 250 & 369 \\
\hline \multirow[t]{2}{*}{$(\mathrm{H}=16 \mathrm{~h})$} & M2 & 3 & 0.156 & 1500.00 & 1414.18 & 24 & 95 & 142 \\
\hline & M1 & 3 & 0.156 & 1500.00 & 1414.18 & 24 & 116 & 159 \\
\hline $5 b$ & SF & 14 & 0.343 & 4500.00 & 4414.80 & 112 & 436 & 651 \\
\hline \multirow[t]{2}{*}{$(\mathrm{H}=32 \mathrm{~h})$} & M2 & 9 & 0.250 & 4500.00 & 4414.80 & 72 & 281 & 424 \\
\hline & M1 & 9 & 0.296 & 4500.00 & 4414.80 & 72 & 332 & 501 \\
\hline $6 a$ & SF & 57 & 1.61 & 25000.00 & 24927.50 & 570 & 2225 & 3354 \\
\hline \multirow[t]{2}{*}{$(\mathrm{H}=144 \mathrm{~h})$} & M2 & 50 & 6.13 & 25000.00 & 24927.50 & 500 & 1952 & 2951 \\
\hline & M1 & 50 & 1.90 & 25000.00 & 24927.50 & 500 & 2310 & 3634 \\
\hline $6 b$ & SF & 111 & 13.84 & 52000.00 & 51933.10 & 1110 & 4331 & 6540 \\
\hline \multirow[t]{2}{*}{$(\mathrm{H}=288 \mathrm{~h})$} & M2 & 104 & 25.72 & 52000.00 & 51933.10 & 1040 & 4058 & 6137 \\
\hline & M1 & 104 & 12.14 & 52000.00 & 51933.10 & 1040 & 4794 & 7576 \\
\hline $6 c$ & SF & 219 & 40.82 & 106000.00 & 105944.00 & 2190 & 8543 & 12912 \\
\hline \multirow[t]{2}{*}{$(\mathrm{H}=576 \mathrm{~h})$} & M2 & 212 & 8.30 & 106000.00 & 105944.00 & 2120 & 8270 & 12509 \\
\hline & M1 & 212 & 27.97 & 106000.00 & 105944.00 & 2120 & 9762 & 15460 \\
\hline 7 & SF & 49 & 33.81 & 21000.00 & 20935.30 & 1176 & 4853 & 8540 \\
\hline \multirow[t]{2}{*}{$(\mathrm{H}=128 \mathrm{~h})$} & M2 & 42 & 43.54 & 21000.00 & 20935.30 & 1008 & 4160 & 7329 \\
\hline & M1 & 42 & 33.59 & 21000.00 & 20935.30 & 1008 & 3724 & 5768 \\
\hline
\end{tabular}

a) $\Delta n=0$ for all cases 
Table 19 Computational results for Examples 8-12 using maximization of productivity as objective ${ }^{\text {a) }}$

\begin{tabular}{|c|c|c|c|c|c|c|c|c|}
\hline Example & Model & Event points & $\begin{array}{l}\text { CPU time } \\
/ \mathrm{s}\end{array}$ & $\begin{array}{c}\text { RMILP } \\
/ \mathrm{cu}\end{array}$ & $\begin{array}{l}\text { MILP } \\
/ \mathrm{cu}\end{array}$ & $\begin{array}{c}\text { Discrete } \\
\text { variables }\end{array}$ & $\begin{array}{c}\text { Continuous } \\
\text { variables }\end{array}$ & Constraints \\
\hline$\overline{8}$ & SF & 5 & 0.109 & 14.00 & 10.00 & 20 & 82 & 117 \\
\hline \multirow[t]{2}{*}{$(\mathrm{H}=6 \mathrm{~h})$} & M2 & 3 & 0.078 & 14.00 & 10.00 & 12 & 40 & 73 \\
\hline & M1 & 3 & 0.062 & 14.00 & 10.00 & 12 & 46 & 79 \\
\hline 9 & SF & 5 & 0.125 & 300.00 & 210.00 & 25 & 114 & 160 \\
\hline \multirow[t]{2}{*}{$(\mathrm{H}=9 \mathrm{~h})$} & M2 & 3 & 0.109 & 300.00 & 210.00 & 15 & 60 & 100 \\
\hline & M1 & 3 & 0.062 & 300.00 & 210.00 & 15 & 80 & 105 \\
\hline 10 & SF & 5 & 0.109 & 80.00 & 58.99 & 30 & 123 & 175 \\
\hline \multirow[t]{2}{*}{$(\mathrm{H}=76 \mathrm{~h})$} & M2 & 2 & 0.125 & 80.00 & 58.99 & 12 & 51 & 73 \\
\hline & M1 & 2 & 0.046 & 80.00 & 58.99 & 12 & 61 & 76 \\
\hline 11 & SF & 6 & 0.109 & 400.00 & 400.00 & 24 & 110 & 153 \\
\hline \multirow[t]{2}{*}{$(\mathrm{H}=10 \mathrm{~h})$} & M2 & 4 & 0.109 & 400.00 & 400.00 & 16 & 74 & 105 \\
\hline & M1 & 4 & 0.093 & 400.00 & 400.00 & 16 & 95 & 129 \\
\hline 12 & SF & 10 & 0.203 & 400.00 & 400.00 & 40 & 182 & 257 \\
\hline \multirow[t]{2}{*}{$(\mathrm{H}=5 \mathrm{~h})$} & M2 & 8 & 0.093 & 400.00 & 400.00 & 32 & 146 & 209 \\
\hline & M1 & 8 & 0.093 & 400.00 & 400.00 & 32 & 183 & 265 \\
\hline
\end{tabular}

a) $\Delta n=0$ for all cases

Table 20 Computational results for Examples 1-3 using minimization of makespan as objective

\begin{tabular}{|c|c|c|c|c|c|c|c|c|}
\hline Example & Model & Event points & $\begin{array}{c}\text { CPU time } \\
/ \mathrm{s}\end{array}$ & $\begin{array}{c}\text { RMILP } \\
/ \mathrm{h}\end{array}$ & $\begin{array}{c}\text { MILP } \\
/ \mathrm{h}\end{array}$ & $\begin{array}{c}\text { Discrete } \\
\text { variables }\end{array}$ & $\begin{array}{c}\text { Continuous } \\
\text { variables }\end{array}$ & Constraints \\
\hline $1 \mathrm{a}(\mathrm{H}=50 \mathrm{~h})$ & SF & 14 & 11.45 & 24.24 & 27.88 & 70 & 268 & 394 \\
\hline \multirow[t]{2}{*}{$D_{s 4}=2000$} & M2 & 12 & 5.30 & 25.36 & 27.88 & 60 & 230 & 342 \\
\hline & M1 & 12 & 6.96 & 24.24 & 27.88 & 60 & 254 & 383 \\
\hline $1 \mathrm{~b}(\mathrm{H}=100 \mathrm{~h})$ & SF & 23 & 7.50 & 48.47 & 52.07 & 115 & 439 & 646 \\
\hline \multirow{2}{*}{$D_{s 4}=4000$} & M2 & 21 & 5.70 & 50.06 & 52.07 & 105 & 401 & 594 \\
\hline & M1 & 21 & 4.81 & 48.47 & 52.07 & 105 & 443 & 671 \\
\hline $2 \mathrm{a}(\mathrm{H}=50 \mathrm{~h})$ & SF & 9 & 96.00 & 10.78 & 19.34 & 72 & 301 & 515 \\
\hline$D_{s 8}=200$ & M2 & 9 & 28.78 & 10.78 & 19.34 & 72 & 301 & 515 \\
\hline$D_{s 9}=200$ & M1 & 9 & 46.58 & 18.68 & 19.34 & 72 & 265 & 425 \\
\hline $2 \mathrm{~b}(\mathrm{H}=100 \mathrm{~h})$ & SF & 19 & $3600^{\text {a) }}$ & 45.57 & 46.31 & 152 & 631 & 1105 \\
\hline$D_{s 8}=500$ & M2 & 19 & $3600^{\mathrm{b})}$ & 45.57 & 46.31 & 152 & 631 & 1107 \\
\hline$D_{s 9}=400$ & M1 & 19 & $3600^{\mathrm{c})}$ & 45.57 & 46.31 & 152 & 555 & 905 \\
\hline $3 \mathrm{a}(\mathrm{H}=50 \mathrm{~h})$ & SF & 7 & 0.187 & 11.07 & 13.37 & 77 & 327 & 572 \\
\hline$D_{s 12}=100$ & M2 & 7 & 0.250 & 11.07 & 13.37 & 77 & 327 & 572 \\
\hline$D_{s 13}=200$ & M1 & 7 & 0.374 & 11.25 & 13.37 & 77 & 306 & 501 \\
\hline $3 b(\mathrm{H}=50 \mathrm{~h})$ & SF & 10 & 0.515 & 12.50 & 17.03 & 110 & 465 & 827 \\
\hline$D_{s 12}=250$ & M2 & 10 & 0.374 & 12.76 & 17.03 & 110 & 465 & 827 \\
\hline$D_{s 13}=250$ & M1 & 10 & 0.359 & 14.27 & 17.03 & 110 & 435 & 723 \\
\hline
\end{tabular}

a) Relative gap $1.10 \%$; b) Relative gap $1.43 \%$; c) Relative gap $1.58 \%$. Note $\Delta n=0$ for all cases

specifically, in Example 1d, the model of Shaik and Floudas [25] requires 9 event points, whereas both models M1 and $\mathbf{M 2}$ require 7 event points, resulting in a reduction in binary variables by $20 \%$ ( 45 vs. 35 ). The optimal schedule for Example 1d using the model M1 is illustrated in Fig. 17.
From Fig. 17, it can be observed that the related production and consumption tasks take place at the same event points because all production tasks in this example are treated as non-recycling tasks. For instance, task I1 processed in unit $\mathrm{J} 1$ is a non-recycling task, which takes place at event point 
N1. Its related consumption task is task I3 processed in unit $\mathrm{J} 3$ since this task $\mathrm{I} 3$ consumes state $\mathrm{S} 2$ produced from task I1, which also takes place at the same event point N1.

Similarly, from Table 18, both mathematical models require 7 event points to generate the optimal solution for Example 4, while the model of Shaik and Floudas [25] requires 10 event points. This leads to $30 \%$ reduction in the number of binary variables (60 vs. 42 ) using the proposed mathematical models. From the optimal schedule for Example 4 using the model M1, which is depicted in Fig. 18, it can be again confirmed that the reduction in the total event points required is due to the fact that all related production and consumption tasks are allowed to take place at the same event points. More specifically, from Fig. 18 , it seems that task $\mathrm{I} 4$ processed in unit $\mathrm{J} 4$, which is a non-recycling, task takes place at event point N3, while its related consumption task is task I6 processed in unit J6 which also takes place at the same event point N3. Briefly, it can be concluded that the proposed models M1 and M2 can be applied to any batch processes even those with recycling loops such as the batch processes in Fig. 1 and Fig. 8.

From Tables 16-17, we can observe that the proposed formulations $\mathbf{M 1}$ and $\mathbf{M} 2$ require the same number of event points with the model of Shaik and Floudas [25] for Examples 2-3. For these examples, M1 and M2 do not reduce the computational time too much because of the same number of event points required. It should be noted though that when tasks have to span over multiple event points, then model M1 can significantly reduce the computational time. This main reason may come from the Constraint (5) which is tighter than those in Shaik and

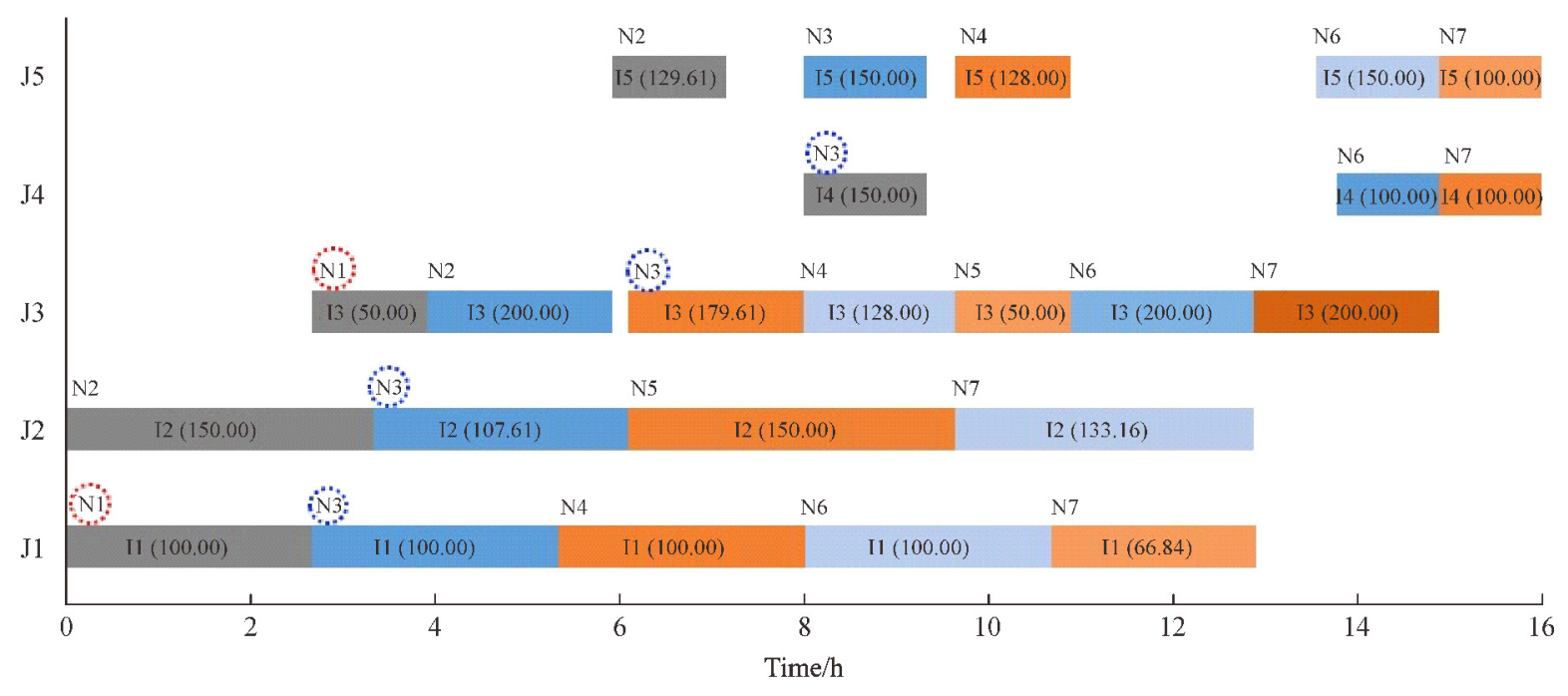

Fig. 17 Optimal schedule of Example 1d using the model M1, with maximization of productivity as the objective.

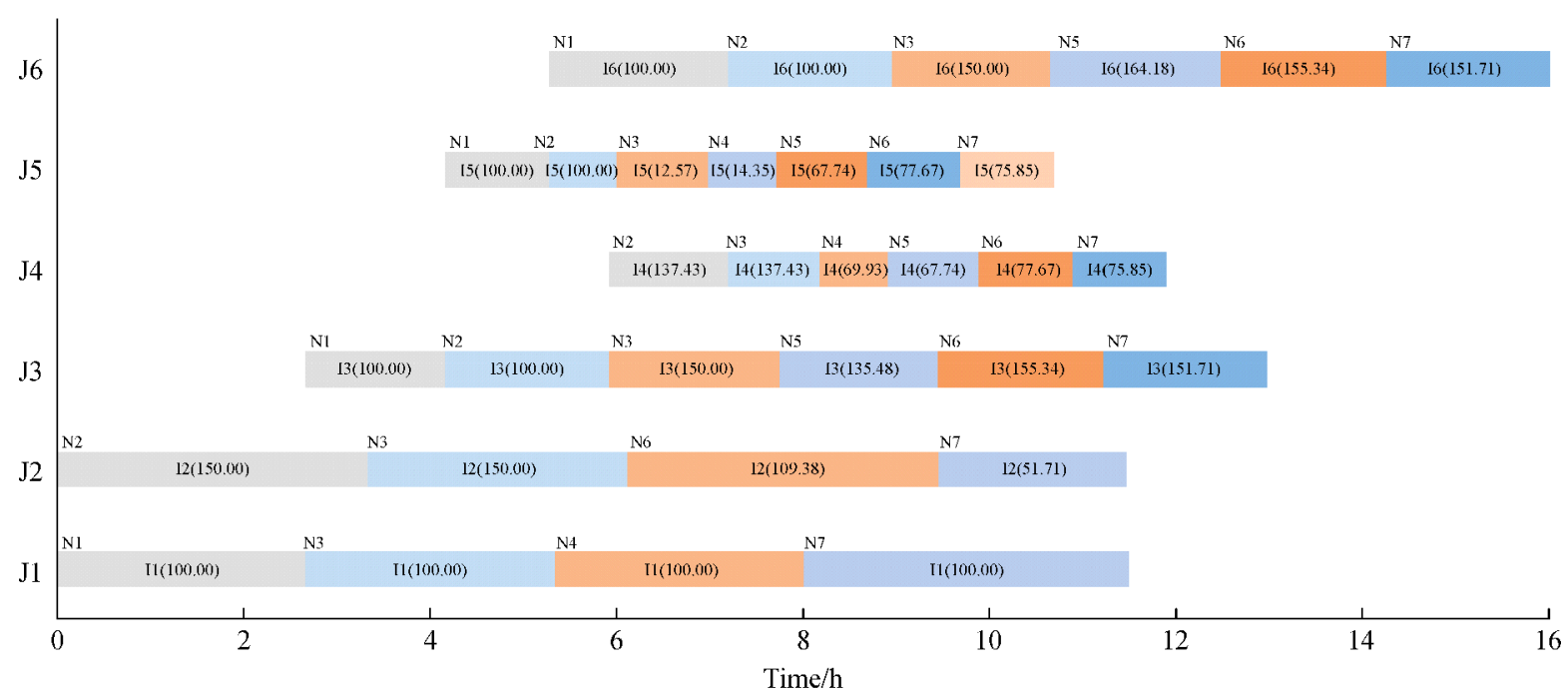

Fig. 18 Optimal schedule for Example 4 using the model M1 with maximization of productivity as the objective. 
Table 21 Computational results for Example 4 using the iterative procedure (maximization of productivity)

\begin{tabular}{|c|c|c|c|c|}
\hline \multirow{3}{*}{ Event point } & \multicolumn{4}{|c|}{$\mathrm{CPU}$ time $/ \mathrm{s}$} \\
\hline & \multicolumn{2}{|c|}{ SF } & \multicolumn{2}{|c|}{ M1 } \\
\hline & $(\Delta n=0)$ & $(\Delta n=1)$ & $(\Delta n=0)$ & $(\Delta n=1)$ \\
\hline$n=1$ & - & - & 0.093 & 0.078 \\
\hline$n=2$ & - & - & 0.062 & 0.078 \\
\hline$n=3$ & - & - & 0.078 & 0.031 \\
\hline$n=4$ & 0.031 & 0.062 & 0.078 & 0.187 \\
\hline$n=5$ & 0.062 & 0.094 & 0.218 & 0.405 \\
\hline$n=6$ & 0.078 & 0.078 & 1.36 & 9.70 \\
\hline$n=7$ & 0.046 & 0.188 & 35.88 & 700 \\
\hline$n=8$ & 0.250 & 0.421 & 532.5 & - \\
\hline$n=9$ & 1.20 & 19.6 & - & - \\
\hline$n=10$ & 24.18 & 2027 & - & - \\
\hline$n=11$ & 698 & - & - & - \\
\hline Total & \multicolumn{2}{|c|}{2771} & \multicolumn{2}{|c|}{1281} \\
\hline
\end{tabular}

Floudas [25] when a task has to span over multiple event points. For instance, M1 requires 49\% less computational time than the model of Shaik and Floudas [25] (5.41 s vs. $2.78 \mathrm{~s}$ ) to solve Example $2 \mathrm{~b}$ and $72 \%$ less computational time ( 3159 s vs. 892 s) for Example 3b. On the other hand, even though $\mathbf{M} 2$ require slightly less computational time than the model of Shaik and Floudas [25] for both Example $2 \mathrm{~b}(5.41 \mathrm{~s}$ vs. $5.10 \mathrm{~s})$ and $3 \mathrm{~b}(3159 \mathrm{~s}$ vs. $3141 \mathrm{~s})$, it requires $46 \%$ more computational time than the model M1 (2.78 s vs. $5.10 \mathrm{~s})$ to solve Example $2 \mathrm{~b}$ and $72 \%$ more computational time (892 s vs. 3141 s) to solve Example $3 \mathrm{~b}$. Therefore, it can be concluded that the proposed model M1 is the most efficient.

However, even though the proposed models, especially model M1, are more efficient for most of the examples, it seems that in some special cases they require a bit larger CPU time. For instance, in Example 4 depicted in Table 18 the model of Shaik and Floudas [25] is able to generate the optimum solution in $24.18 \mathrm{~s}$, while models M1 and M2 require $35.88 \mathrm{~s}$ and $27.63 \mathrm{~s}$ respectively. Nevertheless, the difference is not large, which is in the same magnitude. The main possible reason is that the proposed models M1 and M2 require more CPU time to prove optimality for this example due to different nodes investigated using the branch and bound algorithm. It should also be noted that in Tables 15-20 only the computational time required to generate the optimal solution using the optimum number of event points and $\Delta n$ is reported. In practice, an iterative procedure is often used to find the optimal number of event points, $\Delta n$ and the optimal solution. In this iterative procedure, the problem is solved starting from the minimum number of event points. The number of event points is increased by one until there is no change in the obtained solution. In the iterative procedure, it should be also examined whether allowing one a task to span for more than one event point can lead to the optimal solution. We use the iterative procedure to solve Example 4 with the model of Shaik and Floudas [25] and the proposed model M1. The computational results are given in Table 21. From Table 21 it seems that the model M1 requires less total computational time to locate the optimal solution using the iterative procedure. More specifically for model M1 $1281 \mathrm{~s}$ are required to prove that the optimal solution is generated by using 7 event points while for Shaik and Floudas [25] significantly more time $(2771 \mathrm{~s})$ is required to prove that the optimal solution is generated by using 10 event points.

\section{Conclusions}

In this paper, we proposed two novel mathematical formulations M1 and M2 using the unit-specific eventbased modelling approach. While timing variables in M1 were defined based on units, they were defined based on tasks in M2. In both models, production and consuming tasks related to the same states were allowed to take place at the same event points. To avoid suboptimality in some cases, we proposed a new definition of recycling and nonrecycling tasks. Only the non-recycling production tasks and related consuming tasks are allowed to take place at the same event points. The computational results demonstrate that the proposed models M1 and M2 generated optimal solutions for all examples and reduced the number of event points required, leading to smaller model sizes. Both models are applicable to any batch processes even those with recycling loops. Furthermore, the proposed model M1 is the most efficient since it requires the least possible computational time which can reach up to one 
magnitude in most cases. In the future, we will extend the proposed models $\mathbf{M 1}$ and $\mathbf{M} 2$ to solve other more complex intermediate storage policies such as FIS and NIS.

\footnotetext{
Acknowledgements Nikolaos Rakovitis would like to acknowledge financial support from the postgraduate award by The University of Manchester. Liping Zhang appreciates financial support from the National Natural Science Foundation of China (Grant No. 51875420).
}

Open Access This article is licensed under a Creative Commons Attribution 4.0 International License, which permits use, sharing, adaptation, distribution and reproduction in any medium or format, as long as you give appropriate credit to the original author(s) and the source, provide a link to the Creative Commons licence, and indicate if changes were made. The images or other third party material in this article are included in the article's Creative Commons licence, unless indicated otherwise in a credit line to the material. If material is not included in the article's Creative Commons licence and your intended use is not permitted by statutory regulation or exceeds the permitted use, you will need to obtain permission directly from the copyright holder. To view a copy of this licence, visit http://creativecommons.org/licenses/by/4.0/.

\section{Nomenclature}

Task-specific event-based model

Indices

$i, i^{\prime}:$ tasks

$j_{j} j^{\prime}:$ units

$n, n^{\prime}, n^{\prime \prime}:$ event points

$s:$ states

Sets

$I$ : tasks

$\mathbf{I}_{j}$ : tasks that can be performed in unit $j$

$\mathbf{I}_{S}^{\mathrm{C}}$ : tasks that consume state $s$

$\mathbf{I}_{S}^{\mathrm{P}}$ : tasks that produce state $s$

$\mathbf{I}^{\mathrm{R}}$ : tasks considered as recycling tasks

$J$ : units

$N$ : event points

$S$ : states

$\mathbf{S}^{\mathrm{FP}}$ : states that are final products

$\mathbf{S}^{\mathrm{IN}}$ : states that are intermediate products

$\mathbf{S}^{\mathrm{R}}$ : states that are raw materials

Parameters

$B_{i}^{\max }$ : maximum batch size that can be processed in task $i$

$B_{i}^{\min }:$ minimum batch size that can be processed in task $i$

$D_{S}$ : demand of state $s$

$H$ : scheduling horizon

$p_{s}$ : price of state $s$

$\alpha_{i}$ : coefficient of constant term of processing time of task $i$

$\beta_{i}$ : coefficient of variable term of processing time of task $i$

$\Delta n$ : maximum number of event points that task $i$ is allowed to be active

$\rho_{i, s}:$ portion of state $s$ consumed/produced by task $i$

Binary Variables

$w_{i, n, n^{\prime}}$ : binary variable which takes the value 1 if task $i$ starts at time event point $n$ and finishes at time event point $n^{\prime} \geqslant n$.

Continuous Variables

$b_{i, n, n^{\prime}}$ : batch size of task $i$ that is active from time event point $n$ to time event point $n^{\prime} \geqslant n$

$S T 0_{s}$ : initial amount of state $s\left(s \in S^{R}\right)$

$S T_{s, n}$ : excess amount of state $s$ that needs to be stored at time event point $n$

$T_{i, n}^{\mathrm{f}}$ : finish time of task $i$ at time event point $n$

$T_{i, n}^{\mathrm{s}}$ : start time of task $i$ at time event point $n$

Unit-specific event-based model

Indices

$i, i^{\prime}:$ tasks

$j, j^{\prime}:$ units

$n, n^{\prime}, n^{\prime \prime}:$ event points

$s:$ states

Sets

$I$ : tasks

$\mathbf{I}_{j}$ : tasks that can be performed in unit $j$

$\mathbf{I}_{S}^{\mathrm{C}}$ : tasks that consume state $s$

$\mathbf{I}_{S}^{\mathrm{P}}$ : tasks that produce state $s$

$\mathbf{I}^{\mathrm{R}}$ : tasks considered as recycling tasks

$J$ : units

$N$ : event points

$S:$ states

$\mathbf{S}^{\mathrm{FP}}$ : states that are final products

$\mathbf{S}^{\mathrm{IN}}$ : states that are intermediate products

$\mathbf{S}^{\mathrm{R}}$ : states that are raw materials

Parameters

$B_{i, j}^{\max }$ : maximum batch size of task $i$ processed in unit $j$

$B_{i}^{\text {min }}$ : minimum batch size of task $i$ processed in unit $j$

$D_{S}$ : demand of state $s$

$H$ : scheduling horizon

$p_{s}$ : price of state $s$

$\alpha_{i, j}$ : coefficient of constant term of processing time of task $i$ in unit $j$

$\beta_{i, j}$ : coefficient of variable term of processing time of task $i$ in unit $j$

$\Delta n$ : maximum number of event points that task $i$ is allowed to be active

$\rho_{i, j, s}$ : portion of state $s$ consumed/produced by task $i$ processed in unit $j$

Binary variables

$w_{i, j, n, n^{\prime}}$ : binary variable which takes the value 1 if task $i$ is processed in unit $j$ from time event point $n$ to time event point $n^{\prime} \geqslant n$

Continuous variables

$b_{i, j, n, n^{\prime}}$ : amount of materials that are processed in unit $j$ processing task $i$ from time event point $n$ to time event point $n^{\prime} \geqslant n$

$S T_{s, n}$ : amount of state $s$ that has to be stored at time event point $n$

$T_{j, n}^{\mathrm{s}}$ : start time of unit $j$ at time event point $n$

$T_{j, n}^{\mathrm{f}}$ : end time of unit $j$ at time event point $n$ 
$T_{i, j, n}^{\mathrm{s}}$ : start time of task $i$ in unit $j$ at time event point $n$ $T_{i, j, n}^{\mathrm{f}}$ : end time of task $i$ in unit $j$ at time event point $n$

\section{References}

1. Kondili E, Pantelides C C, Sargent R W H. A general algorithm for short-term scheduling of batch operations-I MILP formulation. Computers \& Chemical Engineering, 1993, 17(2): 211-227

2. Pantelides C. Unified frameworks for optimal process planning and scheduling. Proceedings of the Second Conference on Foundations of Computer Aided Operations, 1994, 253-274

3. Floudas C A, Lin X. Continuous-time versus discrete-time approaches for scheduling of chemical processes: A review. Computers \& Chemical Engineering, 2004, 28(11): 2109-2129

4. Méndez C A, Cerdá J, Grossmann I E, Harjukoski I, Fahl M. Stateof-the-art review of optimization methods for short-term scheduling of batch processes. Computers \& Chemical Engineering, 2006, 30(6-7): 913-946

5. Li J, Susarla N, Karimi I A, Shaik M A, Floudas C A. An analysis of some unit-specific event-based models for the short-term scheduling of noncontinuous processes. Industrial \& Engineering Chemistry Research, 2010, 49(2): 633-647

6. Maravelias C T. General framework and modeling approach classification for chemical production scheduling. AIChE Journal. American Institute of Chemical Engineers, 2012, 58(6): 1812-1828

7. Harjunkoski I, Maravelias C, Bongers $\mathrm{P}$, Castro $\mathrm{P}$, Engell S, Grossmann I, Hooker J, Méndez C, Sand G, Wassick J. Scope for industrial application of production scheduling models and solution methods. Computers \& Chemical Engineering, 2014, 62(5): 161193

8. Velez S, Maravelias C T. Multiple and nonuniform time grids in discrete-time MIP models for chemical production scheduling. Computers \& Chemical Engineering, 2013, 53(11): 70-85

9. Lee H, Maravelias C T. Discrete-time mixed integer programming models for short-term scheduling in multipurpose environments. Computers \& Chemical Engineering, 2017, 107: 171-183

10. Pinto J M, Grossmann I E. A continuous time mixed integer linear programming model for short-term scheduling of multistage batch plants. Industrial \& Engineering Chemistry Research, 1995, 34(9): 3037-3051

11. Sundaramoorthy A, Karimi I A. A simpler better slot-based continuous-time formulation for short-term scheduling in multipurpose batch plants. Chemical Engineering Science, 2005, 60(10): 2679-2702

12. Susarla N, Li J, Karimi I. A novel approach to scheduling multipurpose batch plants using unit-slots. AIChE Journal. American Institute of Chemical Engineers, 2010, 56(7): 1859-1879

13. Zhang X, Sargent R W H. The optimal operation of mixed production facilities-A general formulation and some approaches for the solution. Computers \& Chemical Engineering, 1996, 20(6-7):
897-904

14. Castro P, Barbosa-Póvoa A P F D, Matos H. An improved RTN continuous-time formulation for the short-term scheduling of multipurpose batch plants. Industrial \& Engineering Chemistry Research, 2001, 40(9): 2059-2068

15. Maravelias C T, Grossmann I E. New general continuous-time statetask network formulation for short-term scheduling of multipurpose batch plants. Industrial \& Engineering Chemistry Research, 2003, 42(13): 3056-3074

16. Ierapetritou $M$ G, Floudas $C$ A. Effective continuous-time formulation for short-term scheduling. 1. Multipurpose batch processes. Industrial \& Engineering Chemistry, 1998, 37(11): 4341-4359

17. Li J, Floudas C. Optimal event point determination for short-term scheduling of multipurpose batch plants via unit-specific eventbased continuous-time approaches. Industrial \& Engineering Chemistry Research, 2010, 49(16): 7446-7469

18. Tang Q H, Li J, Floudas C A, Deng M X, Yan Y B, Xi Z H, Chen P $\mathrm{H}$, Kong J Y. Optimization framework for process scheduling of operation-dependent automobile assembly lines. Optimization Letters, 2012, 6(4): 797-824

19. Li J, Xiao X, Floudas C A. Integrated gasoline blending and order delivery operations: Part I. Short-term scheduling and global optimization for single and multi-period operations. AIChE Journal. American Institute of Chemical Engineers, 2016, 62(6): 2043-2070

20. Méndez C A, Cerdá J. Optimal scheduling of a resource-constrained multiproduct batch plant supplying intermediates to nearby endproduct facilities. Computers \& Chemical Engineering, 2000, 24(2-7): 369-376

21. Hui C, Gupta A, van der Meulen H A J. A novel MILP formulation for short-term scheduling of multi-stage multi-product batch plants with sequence-dependent constraints. Computers \& Chemical Engineering, 2000, 24(12): 2705-2717

22. Méndez C A, Cerdá J. An MILP continuous-time framework for short-term scheduling of multipurpose batch processes under different operation strategies. Optimization and Engineering, 2003, 4(1-2): 7-22

23. Li J, Karimi I A. Scheduling gasoline blending operations from recipe determination to shipping using unit slots. Industrial \& Engineering Chemistry Research, 2011, 50(15): 9156-9174

24. Shaik M A, Janak S L, Floudas C A. Continuous-time models for short-term scheduling of multipurpose batch plants: A comparative study. Industrial \& Engineering Chemistry Research, 2006, 45(18): 6190-6209

25. Shaik M, Floudas C. Novel unified modeling approach for shortterm scheduling. Industrial \& Engineering Chemistry Research, 2009, 48(6): 2947-2964

26. Shaik M, Vooradi R. Short-term scheduling of batch plants: Reformulation for handling material transfer at the same event. Industrial \& Engineering Chemistry, 2017, 56(39): 11175-11185 